\title{
Protein kinase A determines platelet life span and survival by regulating apoptosis
}

\author{
Lili Zhao, ${ }^{1}$ Jun Liu, ${ }^{1}$ Chunyan He, ${ }^{2}$ Rong Yan, ${ }^{1}$ Kangxi Zhou, ${ }^{1}$ Qingya Cui, ${ }^{1}$ Xingjun Meng, ${ }^{1}$ Xiaodong Li, ${ }^{1}$ Yang Zhang, ${ }^{1}$ Yumei Nie, ${ }^{1}$ \\ Yang Zhang, ${ }^{1}$ Renping Hu, ${ }^{1}$ Yancai Liu, ${ }^{1}$ Lian Zhao, ${ }^{1,2}$ Mengxing Chen, ${ }^{1}$ Weiling Xiao, ${ }^{1}$ Jingluan Tian, ${ }^{1}$ Yunxiao Zhao, ${ }^{1}$ Lijuan Cao, \\ Ling Zhou, ${ }^{1}$ Anning Lin, ${ }^{3}$ Changgeng Ruan, ${ }^{1}$ and Kesheng Dai ${ }^{1}$ \\ IJiangsu Institute of Hematology, The First Affiliated Hospital and Collaborative Innovation Center of Hematology, Soochow University, Key Laboratory of Thrombosis and Hemostasis, Ministry of Health, \\ Suzhou, China. 'Department of Clinical Laboratory, The Second Affiliated Hospital of Soochow University, Suzhou, China. ${ }^{3}$ Ben May Department for Cancer Research, The University of Chicago, \\ Chicago, Illinois, USA.
}

\begin{abstract}
Apoptosis delimits platelet life span in the circulation and leads to storage lesion, which severely limits the shelf life of stored platelets. Moreover, accumulating evidence indicates that platelet apoptosis provoked by various pathological stimuli results in thrombocytopenia in many common diseases. However, little is known about how platelet apoptosis is initiated or regulated. Here, we show that PKA activity is markedly reduced in platelets aged in vitro, stored platelets, and platelets from patients with immune thrombocytopenia (ITP), diabetes, and bacterial infections. Inhibition or genetic ablation of PKA provoked intrinsic programmed platelet apoptosis in vitro and rapid platelet clearance in vivo. PKA inhibition resulted in dephosphorylation of the proapoptotic protein BAD at Ser155, resulting in sequestration of prosurvival protein BCL-XL in mitochondria and subsequent apoptosis. Notably, PKA activation protected platelets from apoptosis induced by storage or pathological stimuli and elevated peripheral platelet levels in normal mice and in a murine model of ITP. Therefore, these findings identify PKA as a homeostatic regulator of platelet apoptosis that determines platelet life span and survival. Furthermore, these results suggest that regulation of PKA activity represents a promising strategy for extending platelet shelf life and has profound implications for the treatment of platelet number-related diseases and disorders.
\end{abstract}

\section{Introduction}

Platelets are the central regulator for keeping the vital balance between thrombosis and hemorrhage in the circulation and, simultaneously, play essential roles in many important pathophysiological processes, such as atherosclerosis, immune response, infections, and tumor progression and metastasis. However, platelets have very short life spans, and the mystery of platelets present in the circulation for only 8 to 9 days has puzzled human beings for more than half a century (1). More importantly, thrombocytopenia, which can cause life-threatening hemorrhage, often occurs in many common diseases, such as infections $(2,3)$, immune thrombocytopenia (ITP) (4), and diabetes (5), as well as during some pharmacological treatments (6). The pathogenesis of the shortened platelet life span during these different pathological processes is not fully understood. Furthermore, properties of platelets, including short life span and especially the storage lesion, limit the life of stored platelets, which are the best substance for the treatment of thrombocytopenia. Therefore, it is physiologically and pathophysiologically important to find the mechanism for the regulation of platelet life span and survival.

Platelet apoptosis highlighted in recent years sheds light on disclosing the regulatory mechanism of platelet life span and sur-

Authorship note: L. Zhao, J. Liu, C. He, and R. Yan contributed equally to this work. Conflict of interest: The authors have declared that no conflict of interest exists. Submitted: May 15, 2017; Accepted: September 21, 2017.

Reference information: / Clin Invest. 2017;127(12):4338-4351.

https://doi.org/10.1172/JCI95109. vival. Accumulating evidence indicates that the intrinsic program for apoptosis results in platelet destruction under pathological and physiological conditions $(6,7)$. Similarly to eukaryotic cells, BAK and BAX are the 2 fated killers for those platelets that are not consumed during thrombosis and hemostasis $(6,8,9)$. However, among the several prosurvival BCL-2 family proteins, only BCL-XL has been confirmed as interacting with BAK, leading to anuclear platelet apoptosis $(6,8,9)$. Mutations in BCL-XL dosedependently reduced platelet survival, which could be rescued by deletion of BAK and BAX $(6,8)$. In addition, deletion of BAD, a proapoptotic BCL-2 homology domain 3-only (BH3-only) protein, elevated peripheral platelets, although the mechanism was elusive (9). These studies reveal the crucial roles for the apoptotic proteins in the regulation of platelet apoptosis. However, the primary question of how the platelet apoptosis is initiated or suppressed still remains unclear.

PKA is a serine/threonine protein kinase ubiquitous in eukaryotic cells, where it works to control a wide variety of cellular activities, including metabolism, cell growth and differentiation, and gene expression $(10,11)$. PKA activity is regulated by intracellular concentration of cAMP, which is determined by the balance between synthesis and degradation by adenylate cyclase (12) and phosphodiesterases (13), respectively. Both proapoptotic and antiapoptotic effects of PKA were reported in nucleated cells $(14,15)$. PKA is highly expressed, and PKA activity is strictly balanced in platelets $(16,17)$. PKA has been found to play important roles in regulating platelet functions (17). Here, we show that PKA activity is markedly reduced in in vitro-aged platelets or platelets from 


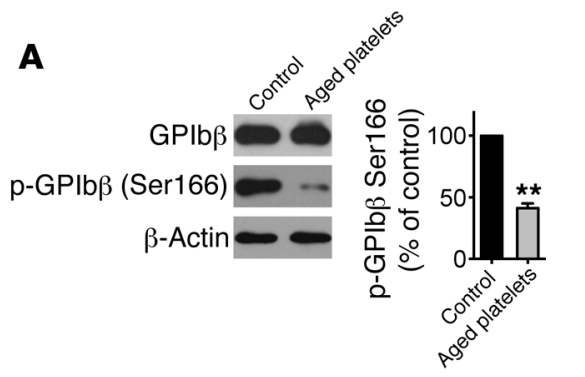

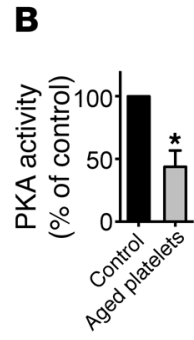

C

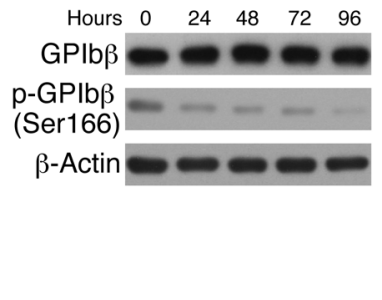

D

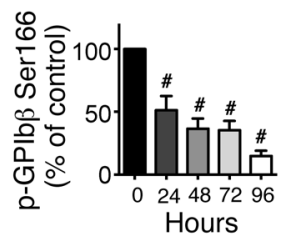

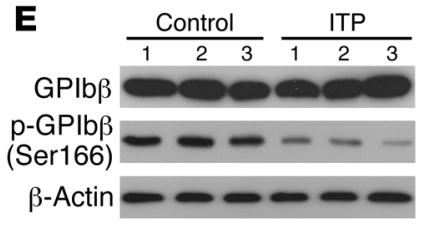

I

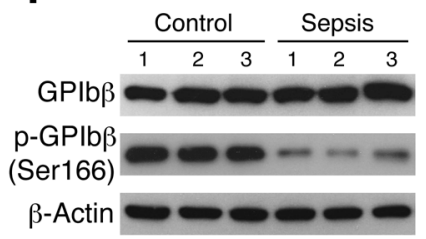

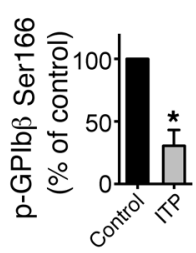

$\mathbf{F}$
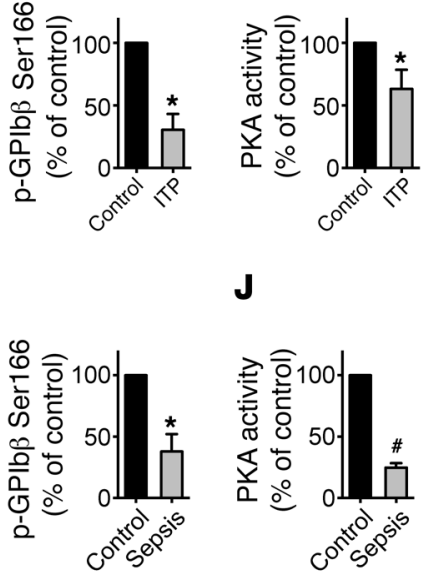

G

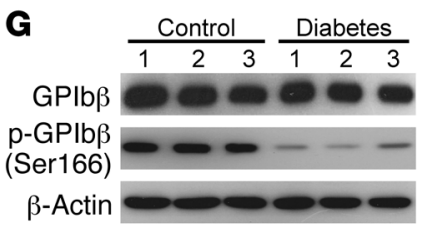

$\beta$-Actin

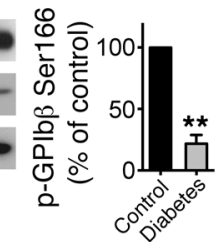

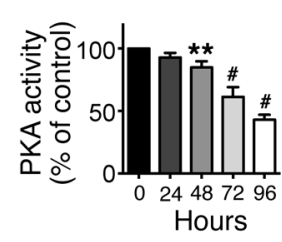

J

$\mathbf{K}$

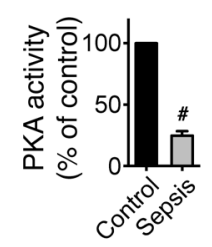

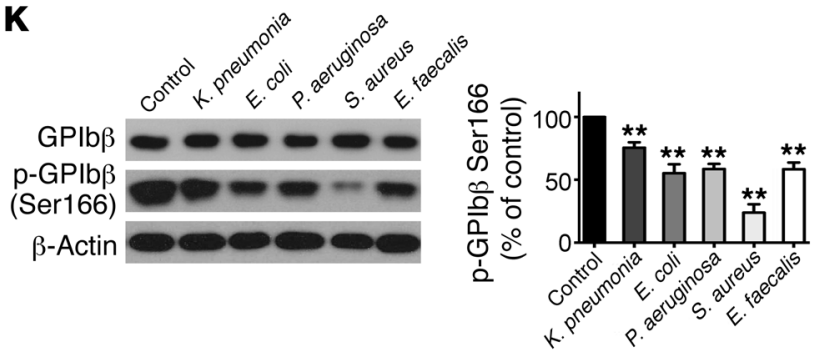

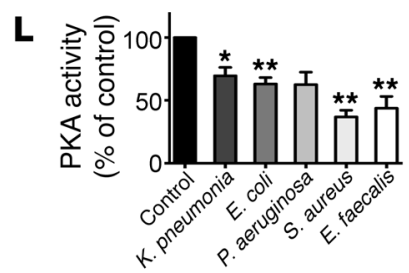

Figure 1. PKA activity is reduced in stored platelets or platelets from patients with ITP, diabetes, and sepsis. (A and B) Western blot analysis of phosphorylated GPIb $\beta$ at Ser166 (pGPIb $\beta$ ) in freshly isolated ( 0 hours, control) or in vitro-aged (16 hours) platelets (left). Densitometry of immunoblots for pGPIb $\beta$ from Western blot data (right) (A). PKA activity in the platelets was examined by ELISA (B). Data are expressed as mean \pm SD from 4 independent experiments. ${ }^{*} P<0.05$; ${ }^{*} P<0.01$, Student's $t$ test. (C and $\mathbf{D}$ ) Washed platelets were incubated at $22^{\circ} \mathrm{C}$ for indicated times. Representative immunoblots and quantification for pGPIb $\beta$ (C) and PKA activity (D) are shown. Data are represented as mean \pm SD from 4 independent experiments. ${ }^{* *} P<0.01$; ${ }^{*} P<$ 0.001 compared with controls ( 0 hours by 1-way ANOVA. (E-J) Platelets were isolated from patients with ITP (E and F), diabetes (G and $\mathbf{H})$, and sepsis (I and $\mathbf{J})$ and age- and sex-matched healthy controls. Representative immunoblots and quantification for pGPIb $\beta$ (E, G, and I) and PKA activity (F, H, and J) are shown. Data in each figure are expressed as mean \pm SD from 6 patients and controls. ${ }^{*} P<0.05 ;{ }^{* *} P<0.01$; ${ }^{*} P<0.001$, compared with controls, Student's $t$ test. (K and $\mathbf{L}$ ) Washed platelets were incubated with indicated bacteria (1:20) or vehicle control at $37^{\circ} \mathrm{C}$ for 90 minutes. Representative immunoblots and quantification for $\mathrm{DGPIb} \beta(\mathbf{K})$ and PKA activity (L) are shown. Data are expressed as mean \pm SD from 4 independent experiments. ${ }^{*} P<0.05$; ${ }^{* *} P<0.01$, compared with control, Student's $t$ test.

patients with ITP, diabetes, and sepsis. Inhibition of PKA causes intrinsically programmed platelet apoptosis in vitro and acute platelet depletion in vivo. More importantly, activation of PKA protects platelets from apoptosis and clearance induced by storage or pathological stimuli. We conclude that PKA is a homeostatic regulator of apoptosis that determines platelet life span and survival under physiological and pathological conditions.

\section{Results}

PKA activity is markedly reduced in in vitro-aged and stored platelets or platelets from patients with ITP, diabetes, and sepsis. Apoptosis delimits platelet life span $(6,8,18)$; however, how apoptosis is reg- ulated remains unclear. We hypothesized that PKA involves regulating platelet apoptosis. Platelet-rich plasma (PRP) was incubated at $37^{\circ} \mathrm{C}$ for 16 hours to generate in vitro-aged platelets $(8,18)$. We found that apoptotic events, consistent with previous reports $(8,18,19)$, were detected in the platelets (Supplemental Figure 1; supplemental material available online with this article; https:// doi.org/10.1172/JCI95109DS1). Simultaneously, PKA activity, as indicated by phosphorylation of PKA substrate GPIb $\beta$ at Ser166 (20) and total PKA activity in the platelets, was obviously reduced in the aged platelets (Figure 1, A and B). Platelets were demonstrated as undergoing apoptosis under blood-banking conditions $(6,8,21)$. We detected apoptotic events in stored platelets (Sup- 
$\mathbf{A}$

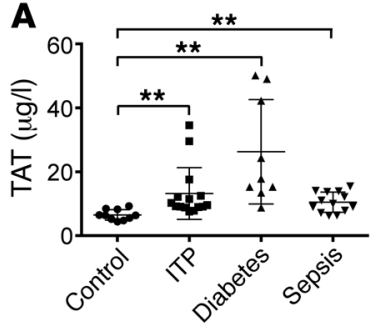

D

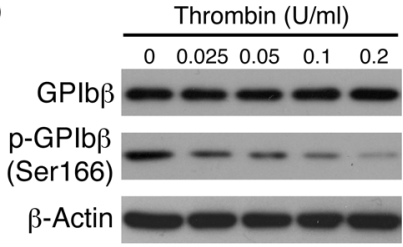

B
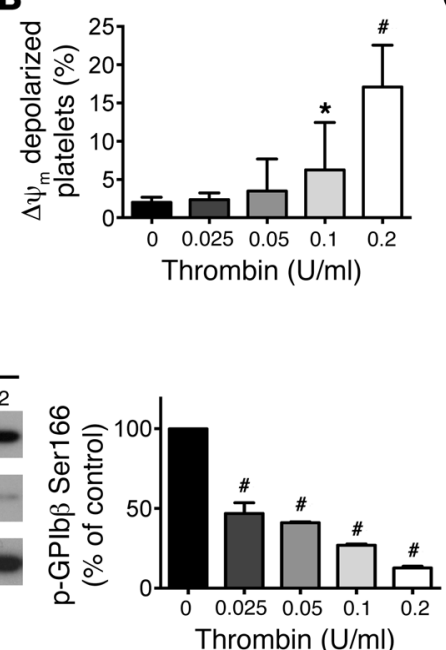

C

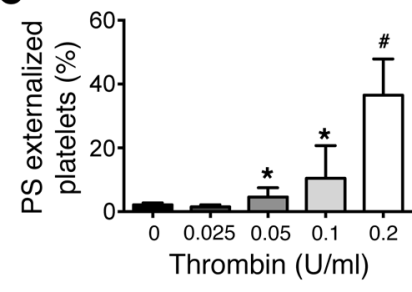

$\mathbf{E}$

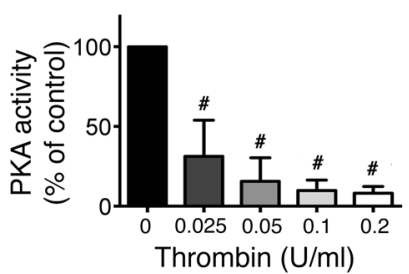

Figure 2. PKA activity in platelets is reduced by thrombin. (A) TAT complexes in plasma from patients with diabetes $(n=9)$, ITP $(n=15)$, and sepsis $(n=13)$ were detected by ELISA. Data are expressed as mean \pm SD. ${ }^{* *} P<0.01$ compared with healthy control, Student's $t$ test. (B-E) Washed platelets were incubated with indicated concentration of thrombin or vehicle (0) at $37^{\circ} \mathrm{C}$ for 30 minutes. Quantifications of $\Delta \psi_{\mathrm{m}}$ depolarization (B) and PS externalization (C) of platelets are shown (mean $\pm S D, n=6$ ). ${ }^{*} P<0.05$; ${ }^{*} P<0.001$ compared with control, 1-way ANOVA. Representative immunoblots and quantification for $p G P I b \beta(D)$ and PKA activity (E) are shown. Data are expressed as mean \pm SD from 6 independent experiments. ${ }^{*} P<0.001$ compared with control, 1-way ANOVA.

levels of thrombin were obviously elevated in the plasma from the 3 kinds of patients (Figure 2A). To further investigate the role of thrombin in regulating PKA activity, different concentrations of thrombin were incubated with platelets. We found that relatively lower concentration of thrombin (less than $0.2 \mathrm{U} / \mathrm{ml}$ ) induced apoptotic events in platelets (Figure 2, $\mathrm{B}$ and $\mathrm{C}$ ) and that PKA activity was dose-dependently reduced by thrombin (Figure 2, D and E). In contrast, PKA activator (forskolin) markedly reduced apoptotic events in platelets stimulated by thrombin (Supplemental Figure 6). Therefore, these data demonstrate the role of thrombin in regulating PKA activity and apoptosis in platelets. However, higher concentration of thrombin (higher than

plemental Figure 1). PKA activity was markedly reduced in a timedependent manner (Figure 1, C and D).

We next examined PKA activity in platelets from patients with thrombocytopenia that occurs in 3 common diseases, ITP, diabetes, and sepsis. As previously reported (2-6), apoptotic events (Supplemental Figure 2) were detected in platelets from these patients (Supplemental Tables 1-3). To our surprise, PKA activity was obviously reduced in all of the platelets from the 3 kinds of patients (Figure 1, E-J). Moreover, incubation of normal platelets with plasma from ITP or diabetes patients incurred platelet apoptosis, and PKA activity was reduced in the platelets simultaneously (Supplemental Figure 3). In contrast, PKA activity was significantly enhanced in platelets stimulated with ristocetin (Supplemental Figure 4).

Recent evidence shows that E. coli and Staphylococcus aureus isolated from sepsis patients induces platelet apoptosis $(2,3)$. To investigate the role of PKA in bacterial infection-induced platelet apoptosis, several common bacteria were incubated with platelets. We found that PKA activity was significantly reduced in all of the platelets (Figure 1, K and L) and that apoptotic events were detected in the platelets synchronously (Supplemental Figure 5). Collectively, these data indicate that PKA activity is reduced in apoptotic platelets induced by different stimulations.

PKA activity in platelets is reduced by thrombin. To explore why PKA activity was reduced in the platelets from different patients, we considered the common pathological stimuli among these diseases. The likeliest candidate is thrombin, since there were reports suggesting that thrombin was generated in the plasma of patients with sepsis (22), diabetes (23), and ITP (24). Furthermore, thrombin was reported to reduce PKA activity by inhibiting adenylate cyclase through $G_{i}(25,26)$ and also activating phosphodiesterase 3A via Akt signaling (26) in platelets. We therefore examined thrombin generation in the plasma and found that the
$0.4 \mathrm{U} / \mathrm{ml}$ ) activated platelets and elevated PKA activity synchronously (Supplemental Figure 7).

Inhibition of PKA incurs intrinsic pathway of platelet apoptosis. Next, we investigated the role of PKA in platelet apoptosis. As previously reported, platelet apoptosis is a mitochondria-mediated intrinsic form of programmed cell death $(6,7)$. Upon apoptotic stimulation, BCL-2 family proteins interact with the mitochondrial outer membrane, leading to transmembrane potential $\left(\Delta \Psi_{\mathrm{m}}\right)$ depolarization as well as activation of caspases $(27,28)$. We found that PKA inhibitor H89 dose-dependently induced $\Delta \Psi_{\mathrm{m}}$ depolarization in platelets (Figure 3A). To exclude nonspecific effects of H89, a PKA-specific inhibitor, Rp-cAMPS, was incubated with platelets, and a similar effect was observed (Supplemental Figure 8). Immunoblots show that H89 activated caspase-3 in a dose-dependent manner (Figure 3B). We examined caspase-3 activity directly and found that caspase- 3 activity was elevated by H89 treatment (Figure 3C). Caspases and other apoptogenic enzymes should disrupt plasma membrane integrity, leading to phosphatidylserine (PS) externalization and typical morphological alterations during apoptosis $(29,30)$. We found that H89 (Figure 3D) and Rp-cAMPS (Supplemental Figure 8) incurred PS externalization. Figure 3E shows that H89 induced platelet shrinkage in a dose-dependent manner. Scanning electron microscope images directly show that H89 dose-dependently induced shrinkage, filopod extrusion, and blebbing of plasma membrane (Figure 3F). H89 also induced apoptotic events in a time-dependent manner (Supplemental Figure 9). In addition, we found that PKA activator forskolin markedly decreased H89-induced platelet apoptotic events (Supplemental Figure 10). Together, these data demonstrate that the PKA inhibitors elicit intrinsic programmed platelet apoptosis.

$P K A$-deficient platelets in conditional PKA C $\alpha-K O$ mice undergo apoptosis. We next confirmed the role of PKA in platelet apop- 

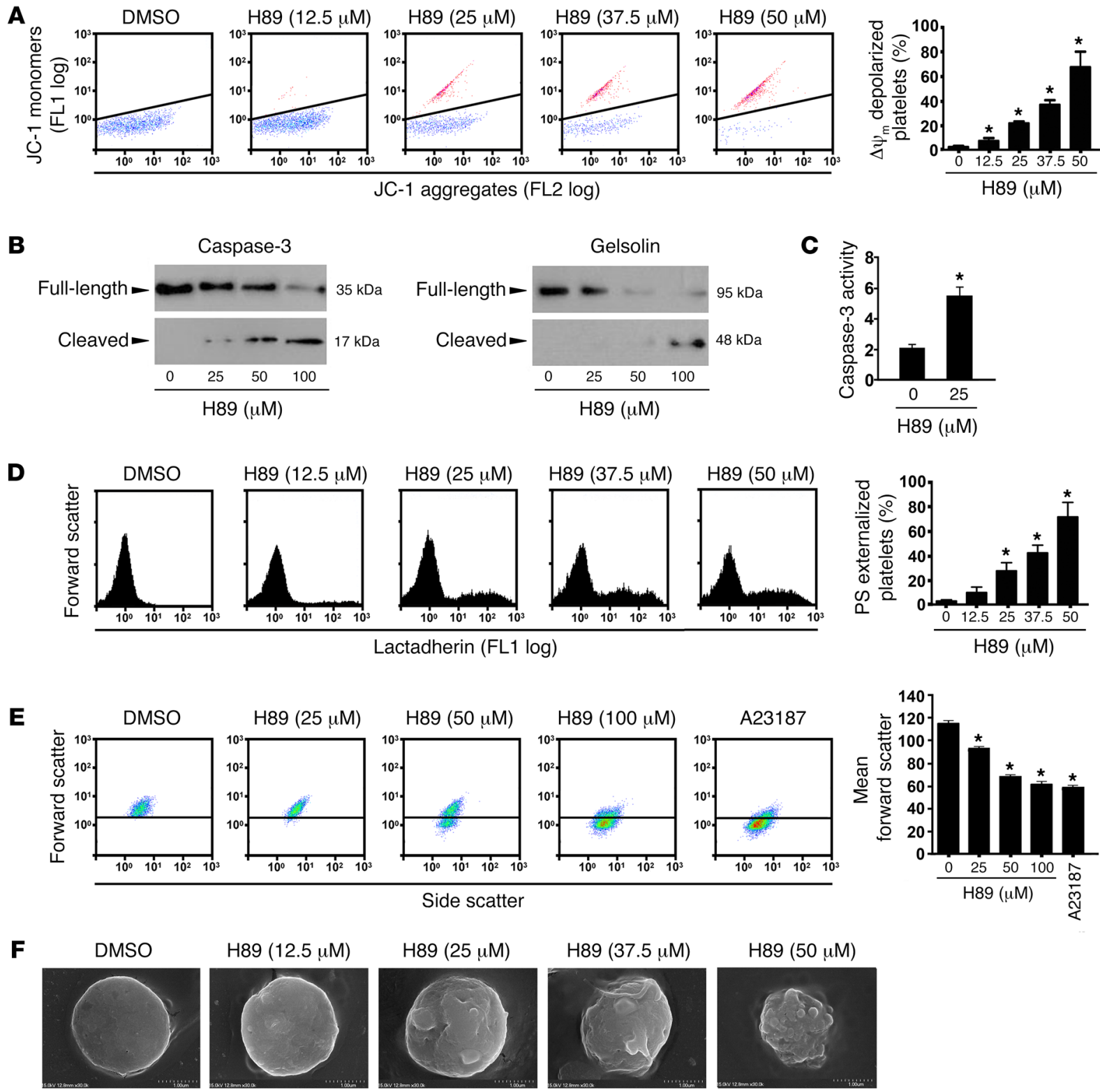

$\mathrm{H} 89(50 \mu \mathrm{M})$

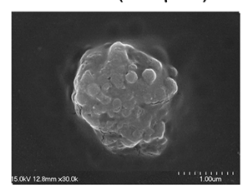

Figure 3. Inhibition of PKA induces platelet apoptosis. (A) Representative flow cytometric figures (left) and quantification (right) of $\Delta \psi_{\mathrm{m}}$ depolarization of platelets incubated with $\mathrm{H} 89$ or vehicle at $22^{\circ} \mathrm{C}$ for 160 minutes (mean $\pm \mathrm{SD}, n=4$ ). ${ }^{*} P<0.05$ compared with control, 1 -way ANOVA. (B and C) Western blot analysis with anti-caspase-3 or anti-gelsolin antibodies (B) and caspase-3 activity analysis (C) in platelets incubated with $\mathrm{H} 89$ or vehicle at $22^{\circ} \mathrm{C}$ for 30 minutes $(n=4) .{ }^{*} P<0.05$ compared with control, Student's $t$ test. (D) Representative flow cytometric figures (left) and quantification (right) of PS externalization of platelets incubated with $\mathrm{H} 89$ or vehicle at $22^{\circ} \mathrm{C}$ for 160 minutes (mean $\pm \mathrm{SD}, n=4$ ). ${ }^{*} P<0.05$ compared with control, 1 -way ANOVA. (E) Representative flow cytometric figures of platelet shrinkage (left) and quantification of mean forward scatter (right) of platelets incubated with $\mathrm{H} 89$, vehicle, or $\mathrm{A} 23187$ at $22^{\circ} \mathrm{C}$ for 30 minutes (mean $\left.\pm \mathrm{SD}, n=4\right) .{ }^{*} P<0.05$ compared with control, 1 -way ANOVA. (F) Representative scanning electron microscopy images of platelets treated with $\mathrm{H} 89$ at $22^{\circ} \mathrm{C}$ for 160 minutes are shown $(n=3)$. Scale bar: $1 \mu \mathrm{m}$.

tosis with genetic ablation mice. Since conventional PKA KO was lethal (11), we generated PF4-Cre-driven megakaryocyte- and platelet-targeted Pkaca (encoding PKA C $\alpha$ ) gene deletion mice (Figure 4A and Supplemental Figure 11). The deficiency of Pkaca and reduced PKA activity detected by dephosphorylated GPIb $\beta$ Ser166 and BAD Ser155 in the platelets were examined by Western blot (Figure 4B). The homozygous Pkaca ${ }^{f / f l} \mathrm{Pf} 4-\mathrm{Cr}^{+}\left(\mathrm{PKA}^{-/-}\right)$, heterozygous Pkacal ${ }^{f /+} \mathrm{Pf} 4-\mathrm{Cr}^{+}\left(\mathrm{PKA}^{-/+}\right)$, and Pkacal ${ }^{\mathrm{fl} / \mathrm{ll}} \mathrm{Pf} 4-\mathrm{Cr} \mathrm{C}^{-}$ $\left(P K A^{+/+}\right.$, expressing normal levels of PKA $\mathrm{C} \alpha$ ) mice did not differ in the number of red and white blood cells and hemoglobin con- centration (Supplemental Table 4). There was not any spontaneous bleeding tendency or thrombotic events over the life spans of $P K A^{-/-}$ and $P K A^{-/+}$mice. We found that $\Delta \Psi_{\mathrm{m}}$-depolarized (Figure $4 \mathrm{C}$ ) and shrunk (Figure 4D) platelets were obviously increased in $P K A^{-/-}$ mice. Scanning electron microscope images illustrate that the $P K A^{-/}$platelets presented shrinkage and blebbing of plasma membrane (Figure $4 \mathrm{E}$ ), indicating that these platelets were undergoing apoptosis. PKA activity and the platelet counts of $P K A^{+/-}$mice were not significantly different from those of $P K A^{+/+}$mice (Figure 4, B and $\mathrm{F}$ ), suggesting that there might be a compensatory mechanism. 
A
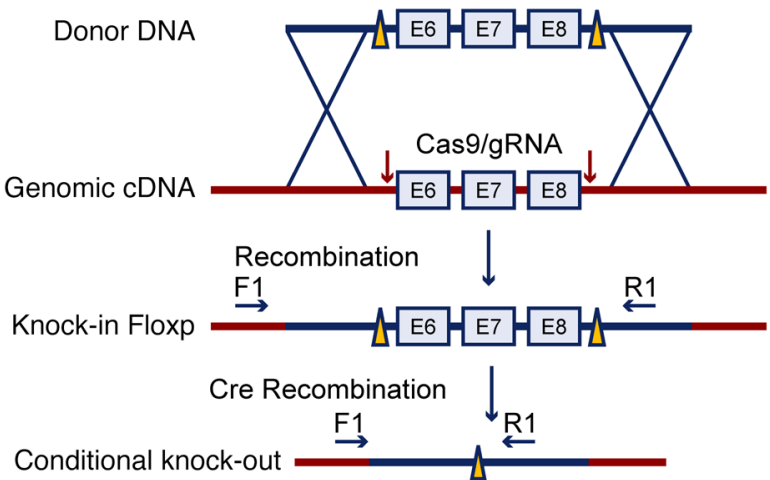

B

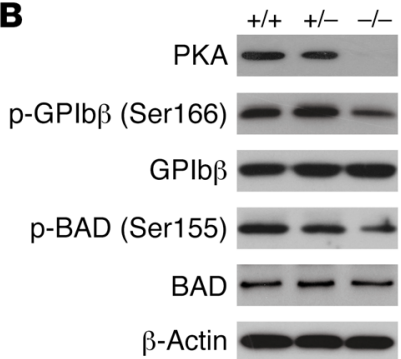

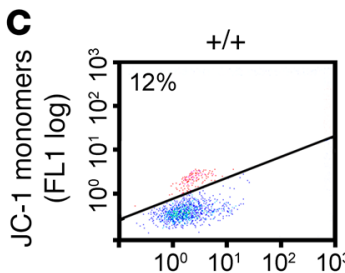
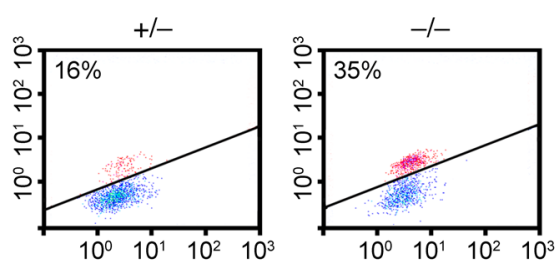

JC-1 aggregates (FL2 log)

\section{D}
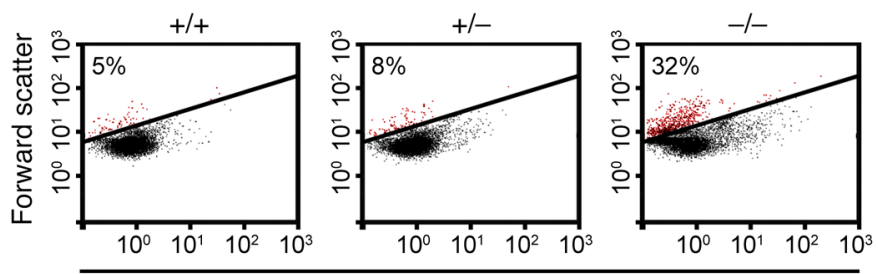

Side scatter
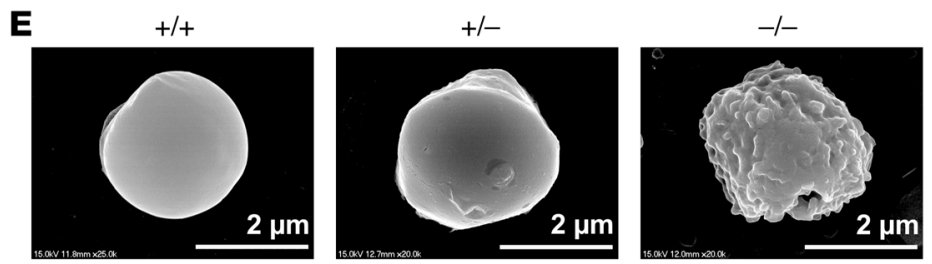

$\mathbf{F}$

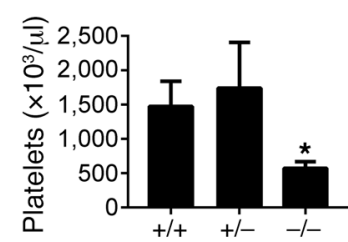

G

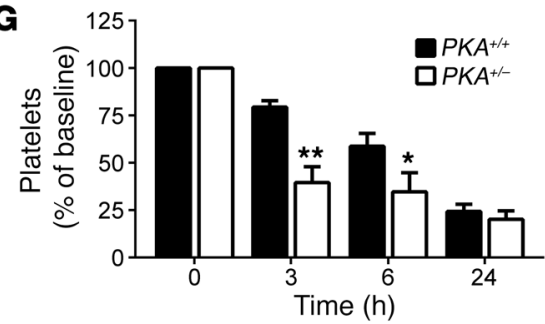

H

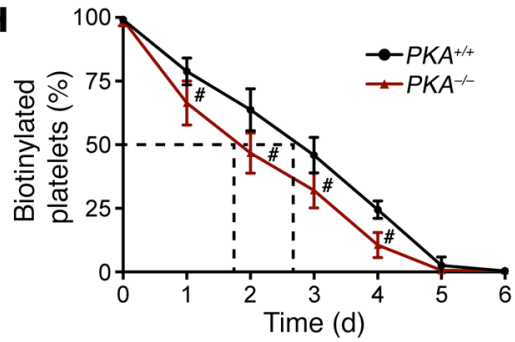

Figure 4. Platelets lacking PKA C $\alpha$ exhibit shortened life span and apoptosis. (A) A PKA C $\alpha$ conditional KO mouse was generated by introducing a loxP site on either side of exons 6, 7, and 8 of the gene $\left(P k a c a^{f l / f}\right)$. Mice with KO specific to megakaryocytes and platelets were produced by crossing the PKA C $\alpha$ floxed mice with transgenic mice expressing PF4 promoter-driven Cre recombinase (Pf4-Cre+). (B) Western blot analysis of PKA C $\alpha$, BAD, GPIb $\beta$, phosphorylated BAD at Ser155, and phosphorylated GPIb $\beta$ at Ser166 in platelets from $P K A^{-/-}, P K A^{-/+}$, and $P K A^{+/+}$mice. The immunoblots shown are representative of at least 5 mice of each genotype. (C-E) Platelets from $P K A^{-/-}, P K A^{+/-}$, and $P K A^{+/+}$mice were analyzed for $\Delta \psi_{\mathrm{m}}$ depolarization (red population) (C), shrinkage (red population) (D), and morphology by scanning electron microscopy (E). Plots or images shown in each panel are representative of at least 3 mice. Scale bars: $2 \mu \mathrm{m}$. (F) Automated analysis of platelet counts. Data are represented as mean $\pm \mathrm{SD}$ of $7 P K A^{+/+}, 7 P K A^{+/-}$, and $5 P K A^{-/-}$male mice. ${ }^{*} P<$ 0.05 , compared with $P K A^{+/+}$mice, Student's $t$ test. (G) Anti-mouse platelet antibody R300 $(0.15 \mathrm{mg} / \mathrm{kg})$ was intraperitoneally injected into $P K A^{+/+}$or $P K A^{+/-}$ mice. Platelet counting was performed at indicated time points. Data are represented as mean \pm SD of $6 P K A^{+/+}$and $6 P K A^{+/-}$mice. ${ }^{*} P<0.05 ;{ }^{* *} P<0.01$, compared with $P K A^{+/+}$mice, 2-way ANOVA. (H) The life span of $P K A^{-/-}$platelets is obviously decreased. Mice were intravenously injected with NHS-biotin. Peripheral blood samples were taken from $P K A^{+/+}$and $P K A^{-/-}$mice 1, 2, 3, 4, 5, and 6 days after injection. The percentage of biotinylated platelets was determined by flow cytometry. Data are represented as mean \pm SD of 5 mice at each time point. ${ }^{P} P<0.001,2$-way ANOVA.

However, injection of anti-platelet mixture antibody R300, which incurs platelet apoptosis (Supplemental Figure 12), induced more rapid platelet clearance in $P K A^{+/-}$mice than in $P K A^{+/+}$mice (Figure $4 \mathrm{G}$ ), suggesting that the $P K A^{+/-}$platelets are more prone to apoptosis. It has been well established that apoptotic cells are rapidly cleared by phagocytes in vivo $(31,32)$. We found that the number of circulatory platelets was markedly reduced in the $P \mathrm{KA}^{-/-}$mice (Fig- ure $4 \mathrm{~F}$ ) and the life span of $P K A^{-/-}$platelets was obviously decreased (Figure $4 \mathrm{H}$ ). However, the proportion of reticulated platelets was not significantly reduced in the $P K A^{-/-}$mice (Supplemental Figure 13). Moreover, the number and morphology of megakaryocytes were not varied in the bone marrow of $P K A^{-/-}$mice compared with that of the WT mice (Supplemental Figure 14). These data suggest that the reduced platelets resulted from apoptosis-induced platelet 
A

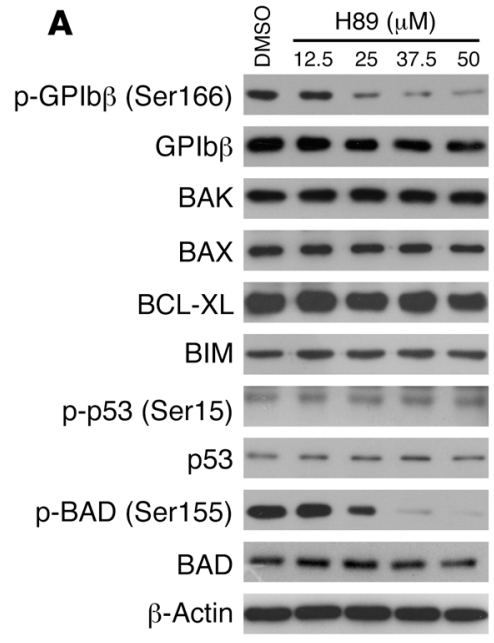

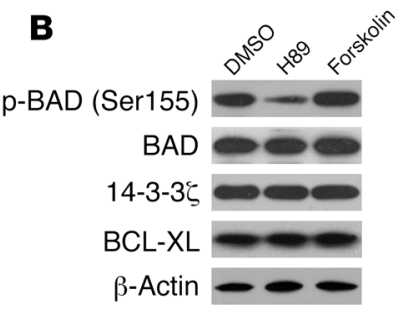

C

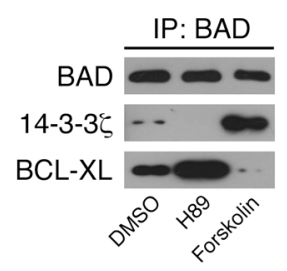

D

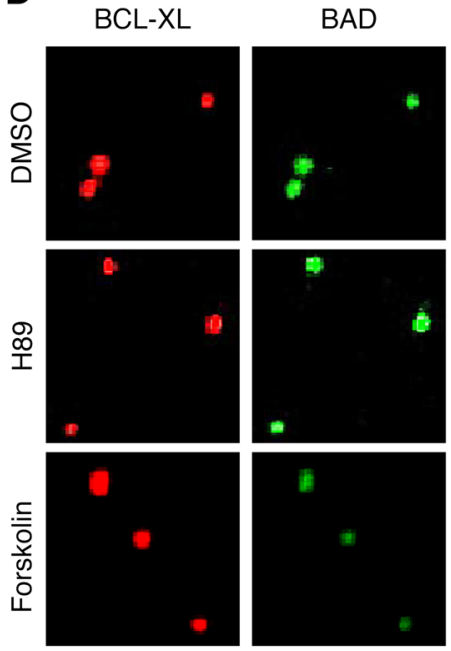

BCL-XL/BAD
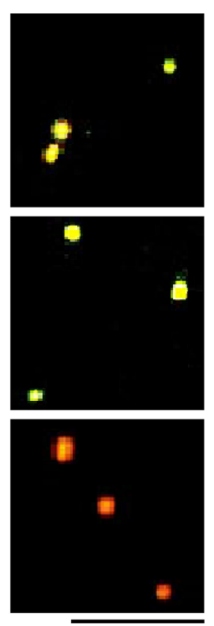

E

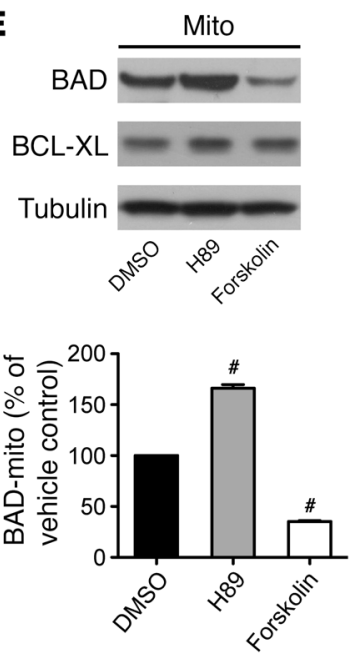

F Blood flow $\square$

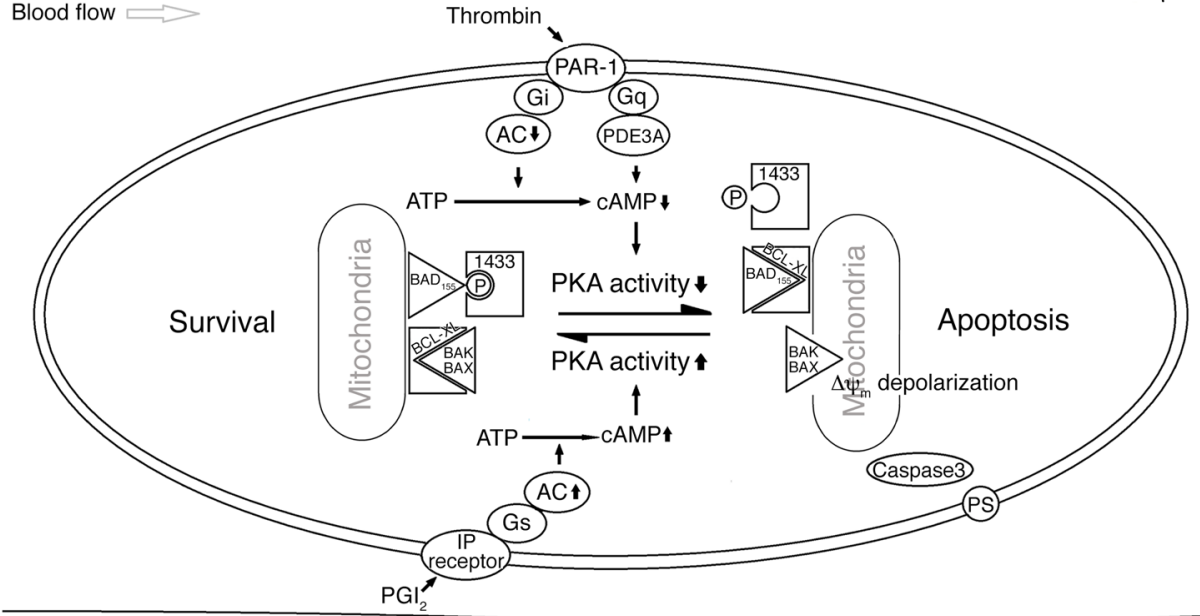

Endothelial cells

Endothelial cells

Figure 5. PKA regulates platelet apoptosis via mediation of phosphorylation of BAD at Ser155. (A) Washed platelets were incubated with indicated concentrations of $\mathrm{H} 89$ or vehicle at $22^{\circ} \mathrm{C}$ for 160 minutes. Western blot analysis for the levels of indicated proteins with different antibodies. (B and C) Washed platelets were incubated with $\mathrm{H} 89(25 \mu \mathrm{M})$, forskolin $(10 \mu \mathrm{M})$, or vehicle at $22^{\circ} \mathrm{C}$ for 120 minutes. The platelets were lysed and centrifugated. Western blot analysis for the levels of indicated proteins with different antibodies (B). The lysates were immunoprecipitated with anti-BAD antibody overnight. Western blot analysis for BAD, BCL-XL, and 14-3-3 with various antibodies (C). (D and E) Washed platelets were incubated with H89 (25 $\mu$ M), forskolin (10 $\mu \mathrm{M}$ ), or vehicle control at $22^{\circ} \mathrm{C}$ for 120 minutes. The mitochondria of the pretreated platelets were isolated and verified as described in Methods. Representative images of mitochondria stained for BAD and BCL-XL are shown. Scale bar: $5 \mu \mathrm{m}$ (D). Western blot analysis for BAD and BCL-XL in the mitochondria, and quantification of the levels of BAD and BCL-XL from the Western blot data (E). Data are expressed as mean $\pm S D(n=5)$. ${ }^{*} P<0.001,1-$ way ANOVA. The blots and images shown in each panel are representative of at least 5 separate experiments with different donors. (F) Schematic representation of proposed mechanism for PKA-regulated platelet apoptosis. Thrombin generated in the circulation (or in the absence of endothelium-derived PGI) reduces PKA activity. The decrease of PKA activity results in dephosphorylation of BAD at Ser155, which releases 14-3-3 and sequesters prosurvival BCL-XL on mitochondria, leading to apoptosis.

destruction. Collectively, these data further demonstrate that $P K A$ deficiency incurs platelet apoptosis.

PKA regulates platelet apoptosis via mediation of BAD Ser155 phosphorylation. We then explored the mechanism for PKA inhibition induced platelet apoptosis. PKA activity was reduced by the PKA inhibitor H89, as indicated by dephosphorylation of GPIb $\beta$ Ser166 (Figure 5A). The levels of apoptotic executors BAK and BAX and the main prosurvival protein BCL-XL were not altered in H89-treated platelets (Figure 5A). PKA was reported to reduce apoptosis via elevation of BIM expression $(15,33)$. We found that the level of BIM was not obviously varied in the PKA inhibitortreated platelets (Figure 5A). There is evidence that PKA inhibition promotes p53 expression and stability (34) and that phosphorylation of p53 inactivates BCL-XL, leading to platelet apoptosis (5, 35). However, neither p53 nor phosphorylated p 53 was altered in the PKA inhibitor-treated platelets (Figure 5A).

We found that phosphorylation of BAD at Ser155 was dosedependently reduced by the PKA inhibitor (Figure $5 \mathrm{~A}$ ). In contrast, the PKA activator forskolin enhanced BAD Ser155 phosphorylation (Figure 5B). Previous studies suggested that BAD Ser155 was 

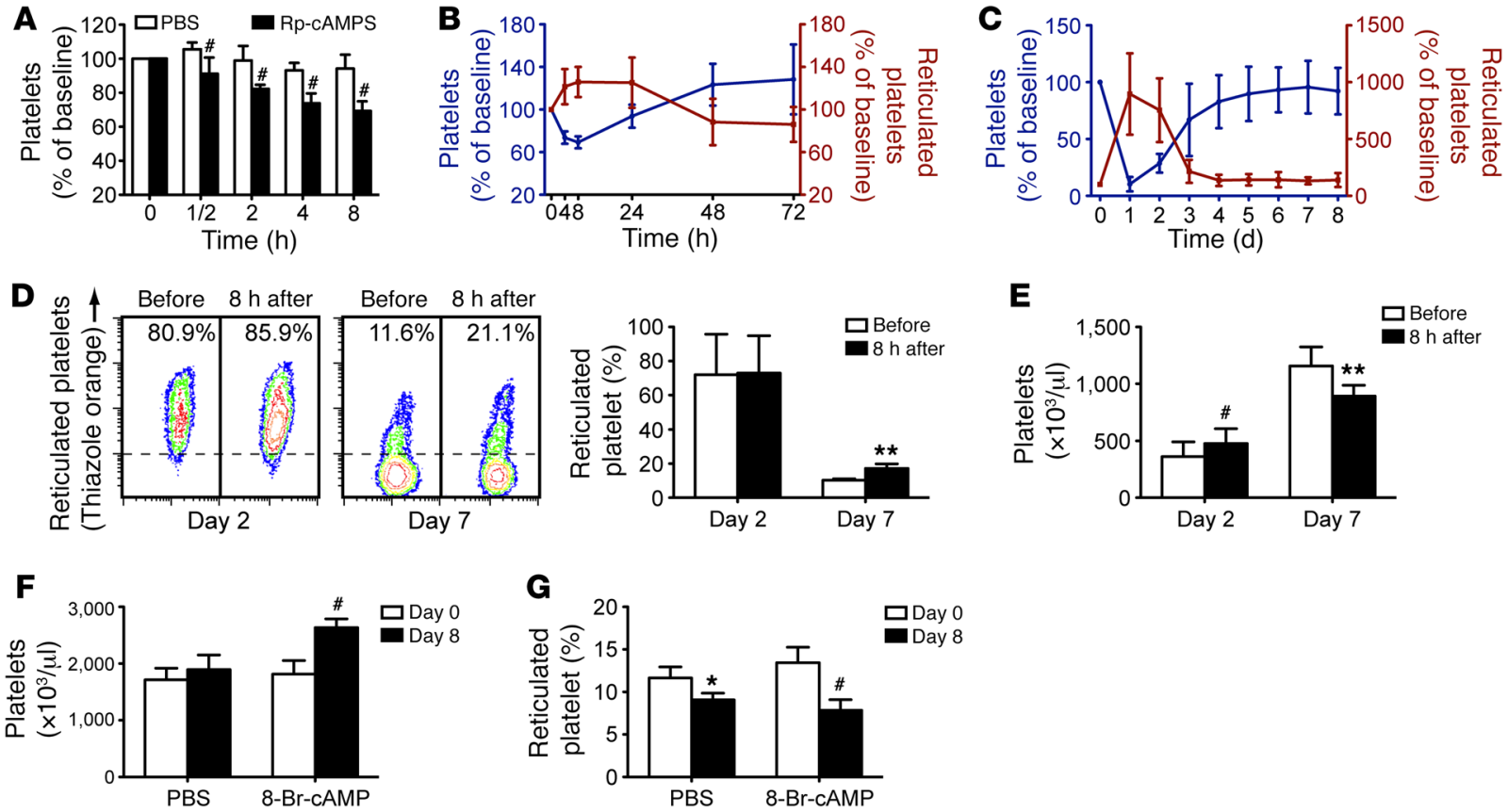

Figure 6. PKA inhibitor causes acute thrombocytopenia, and PKA activator elevates peripheral platelets. (A and B) Male ICR mice were injected with a single dose of Rp-cAMPS $(50 \mathrm{mg} / \mathrm{kg}$ ) through the tail vein. Platelet counts (A and $\mathbf{B})$ and reticulated platelet counts (B) were determined at the indicated time points. ${ }^{\#} P<0.001$, compared with control, 2-way ANOVA. (C) Male ICR mice were intraperitoneally injected with a single dose of anti-platelet antibody $(R 300,0.15 \mathrm{mg} / \mathrm{kg}$ ) to synchronize platelet production. The antibody induced severe thrombocytopenia. The newly synthesized platelets were released into the circulation, as indicated by reticulated platelets (right axis) increasing. The number of platelets (left axis) became stable from day 4 . (D and E) Male ICR mice were intraperitoneally injected with a single dose of anti-platelet antibody (R300, $0.2 \mathrm{mg} / \mathrm{kg}$ ) and then injected with Rp-cAMPS (50 $\mathrm{mg} / \mathrm{kg}$ ) through the tail vein 2 or 7 days afterwards. Reticulated platelet counts (D) and platelet counts (E) were determined prior to or 8 hours after injection of Rp-cAMPS. The left panels show representative flow cytometric profiles of thiazole-orange-stained platelets prior to or 8 hours after Rp-cAMPS injection. (F and $\mathbf{G}$ ) Male ICR mice were injected daily with PKA activator 8-Br-CAMP ( $2.5 \mathrm{mg} / \mathrm{kg}$ ) or vehicle control (PBS) through the tail vein. Platelet counts $(\mathbf{F})$ and reticulated platelet counts $(\mathbf{C})$ were determined at day 8. Data in each panel represent mean \pm SD of 5 to 6 mice at each time point. ${ }^{*} P<0.05 ;{ }^{* *} P<0.01 ;{ }^{\#} P<0.001$, compared with control, Student's $t$ test (D-G).

phosphorylated by PKA, which prevents it from binding to BCL$\mathrm{XL}$ via enhancement of $14-3-3$ association $(36,37)$. We found that the association of BAD with BCL-XL was markedly enhanced or reduced by $\mathrm{H} 89$ or forskolin, respectively. In contrast, binding of 14-3-3 with BAD was reduced by $\mathrm{H} 89$ and increased by forskolin, in contrast with what occurred with BCL-XL (Figure 5C). These data suggest that the PKA inhibitor H89 (or forskolin) inactivates (or activates) prosurvival BCL-XL via regulation of the association of BAD with BCL-XL. Using confocal microscopy, we demonstrate that BAD colocalized with BCL-XL to mitochondria (Figure 5D). H89 enhanced, but forskolin reduced, localization of BAD to mitochondria (Figure 5, D and E). As is known, nonphosphorylated BAD heterodimerized with BCL-XL on mitochondria, thereby releasing the proapoptotic BAX and leading to apoptosis (36-39). Therefore, our results suggest that PKA inhibition results in dephosphorylation of BAD at Ser155, which sequesters prosurvival BCL-XL on mitochondria, leading to apoptosis (Figure 5F). In support of this model, we found that PKA inhibitor H89-induced apoptotic events were markedly decreased in Bad-deficient platelets that lack the PKA substrate (Supplemental Figure 15).

PKA inhibitor causes acute thrombocytopenia. PKA inhibition results in platelet apoptosis in vitro. Thus, we reasoned that PKA inhibitor may shorten platelet life span in vivo. As expected, after intravenously injecting a single dose of PKA inhibitor Rp-cAMPS, platelet counts began to drop in 30 minutes and dropped to a nadir of around $70 \%$ at 8 hours (Figure 6A). Rp-cAMPS caused thrombocytopenia in a dose-dependent manner (data not shown). However, compared with those in WT mice, peripheral platelets were markedly elevated in the $P K A^{-/}$mice treated with Rp-cAMPS (Supplemental Figure 16). Platelet counts began to recover after 8 hours and reached normal levels at around 36 hours after injection (Figure 6B). These data indicate that reduction of PKA activity shortens the life span of peripheral platelets. In addition, Bad deficiency rescued Rp-cAMPS-induced platelet clearance (Supplemental Figure 17), further demonstrating that PKA regulates platelet life span via BAD-mediated platelet apoptosis.

Interestingly, with the decrease of platelet counts, reticulated platelets began to increase (Figure 6B), suggesting that the younger platelets are resistant to PKA inhibitor-induced apoptosis. This finding is consistent with the data showing that apoptosis occurred in aged or stored platelets (Figure 1, A and B). To further investigate whether the older platelets are more susceptible to PKA inhibition-induced clearance in vivo, anti-platelet mixed antibodies (R300), which incur platelet clearance in mice (40), were injected into mice to artificially synchronize platelet production. Almost no platelets were detected in peripheral blood after 1 day of R300 injection, and the platelet numbers recovered within 7 days (Figure 6C). During this period, reticulated platelets increased sharply 
A

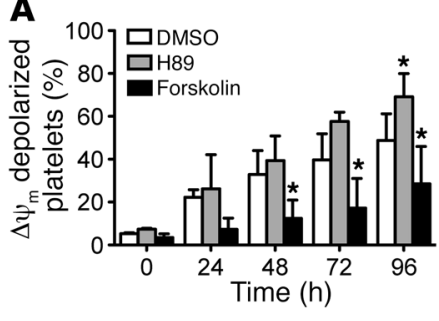

B

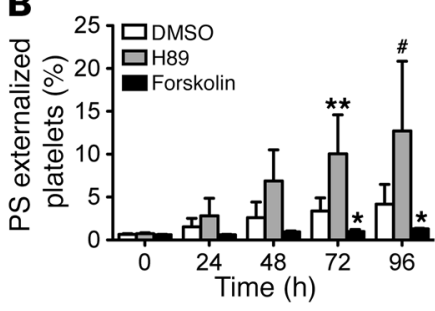

C

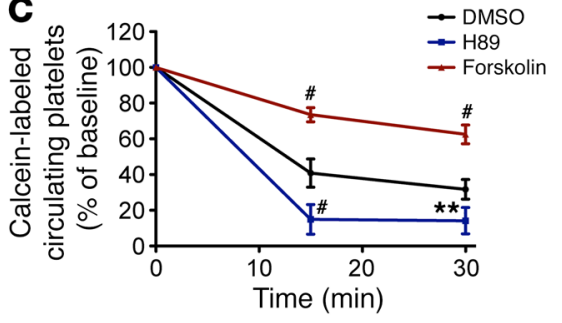

D Ristocetin $(1.25 \mathrm{mg} / \mathrm{ml})$

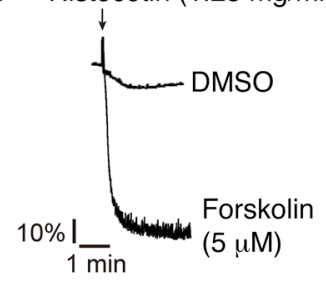

$\mathbf{F}$

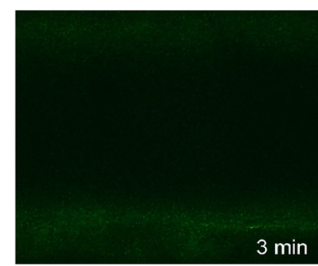

DMSO

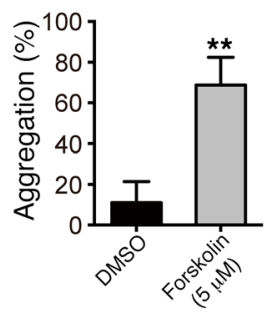

E Collagen $(5 \mu \mathrm{g} / \mathrm{ml})$
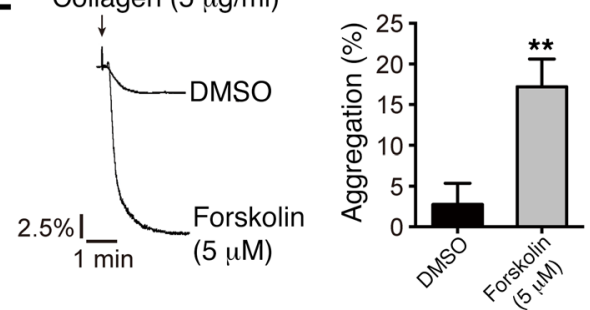

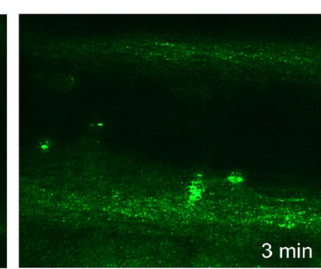

Forskolin

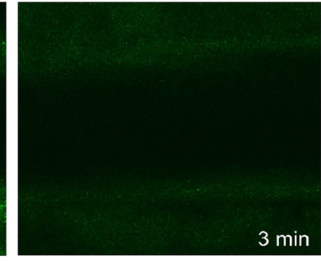

H89

Figure 7. Activation of PKA protects stored platelets from apoptosis, clearance, and loss of function. (A and B) $\Delta \psi_{\mathrm{m}}$ depolarization and PS exposure of human platelets incubated with $\mathrm{H} 89(25 \mu \mathrm{M})$, forskolin $(5 \mu \mathrm{M})$, or vehicle at $22^{\circ} \mathrm{C}$ for indicated times. Data are represented as mean \pm SD from 4 independent experiments. ${ }^{*} P<0.05$; ${ }^{* *} P<0.01$; ${ }^{*} P<0.001$, 2-way ANOVA. (C) Mouse platelets were incubated with H89 (25 $\left.\mu \mathrm{M}\right)$, forskolin $(5 \mu \mathrm{M})$, or vehicle at $22^{\circ} \mathrm{C}$ for 72 hours. The platelets were labeled with calcein and injected into ICR mice $(n=5)$. The percentage of calcein-labeled platelets remaining in circulation was determined by flow cytometry. Data are represented as mean of normalized percentage of calcein-labeled platelets (time point $0=100 \%) \pm$ SD of 3 independent experiments. ${ }^{*} P<0.01 ;{ }^{*} P<0.001$, 2-way ANOVA. (D and E) Human washed platelets were incubated with forskolin ( $\left.5 \mu \mathrm{M}\right)$ or vehicle (DMSO) at $22^{\circ} \mathrm{C}$ for 72 hours. The pretreated washed platelets were stimulated with ristocetin $(1.25 \mathrm{mg} / \mathrm{ml})$ plus von Willebrand factor $(7.5 \mu \mathrm{g} / \mathrm{ml})(\mathbf{D})$ and collagen $(5 \mu \mathrm{g} / \mathrm{ml})(\mathbf{E})$ at $37^{\circ} \mathrm{C}$ under constant stirring. Platelet aggregation was recorded in a CHRONO-LOC aggregometer. Histograms of maximal platelet aggregation under the indicated conditions are shown as mean \pm SD of 5 independent experiments. ${ }^{* *} P<0.01$, Student's $t$ test. (F) Washed mouse platelets were incubated with forskolin $(5 \mu \mathrm{M})$ or vehicle (DMSO) at $22^{\circ} \mathrm{C}$ for 72 hours and were labeled with calcein-AM $(5 \mu \mathrm{g} / \mathrm{ml})$. The recipient mice were injected intravenously with pretreated platelets $\left(5 \times 10^{6} / \mathrm{g}\right)$. $\mathrm{FeCl}_{3}$-induced thrombosis in the mice was recorded by real-time microscopy at 3 minutes. Only forskolin-treated calcein-AM-labeled platelets were detected in the thrombus. No H89-treated or vehicle control-treated (DMSO) stored platelets were found in the thrombus. Each image is representative of 5 mice. Original magnification $\times 200$ ( $\times 10$ eyepiece, $\times 20$ objective).

at the beginning and recovered to normal proportions in around 4 days (Figure 6C). The platelet life span is 5 days in mice. Therefore, PKA inhibitor Rp-cAMPS was injected into the mice on day 2 or 7 after R300 injection, times at which most of the platelets were either young (day 2) or old (day 7) (Figure 6D). Rp-cAMPS destroyed $30 \%$ of peripheral platelets at day 7 ; however, the platelet counts were not reduced by Rp-cAMPS at day 2 (Figure 6E). These data confirm that the older platelets are more susceptible to PKA inhibitor-induced apoptosis and clearance.

PKA activator elevates peripheral platelets. Activation of PKA enhances BAD Ser155 phosphorylation, resulting in inhibition of apoptosis. Therefore, the PKA activator should prevent older platelets from apoptosis and extend platelet life span. To test this hypothesis, PKA activator 8-Br-cAMP was injected into mice daily for 8 days. Platelet counts were increased more than $60 \%$ on day 8 by 8 -Br-cAMP (but not vehicle) (Figure $6 \mathrm{~F}$ ). In contrast, the percentage of reticulated platelets dropped to $60 \%$ of normal levels (Figure 6G), suggesting that the increased platelets were not the result of the generation of new platelets. These data demonstrate that activation of PKA protects platelets from apoptosis and extends platelet life span in vivo.

Activation of PKA protects stored platelets from apoptosis, clearance, and loss of function. Apoptosis results in storage lesion, leading to dysfunction and rapid clearance of transfused platelets $(6,8$, 21). We therefore investigated the role of PKA in regulating platelet apoptosis during storage with PKA activator forskolin and inhibitor H89. To avoid the inhibitory effects of the PKA activator on platelet functions, we performed a dose-dependent experiment, and 5 $\mu \mathrm{M}$ forskolin, which did not significantly inhibit platelet functions (Supplemental Figure 18), was selected. After incubating with stored platelets, H89 promoted apoptosis. In contrast, forskolin markedly reduced apoptotic events in stored platelets (Figure 7, A and B). Mitochondrial $\Delta \Psi_{\mathrm{m}}$ depolarization-mediated intrinsic apoptosis is an irreversible process. These data verify the key role for PKA in regulating platelet apoptosis and also suggest that PKA acts upstream of $\Delta \Psi_{\mathrm{m}}$ depolarization to regulate apoptosis. 
A
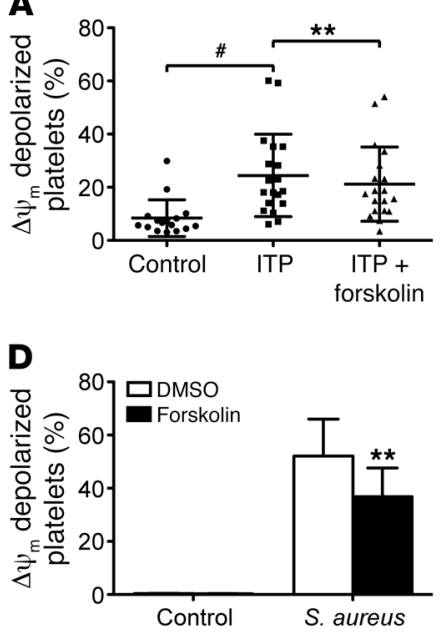

B

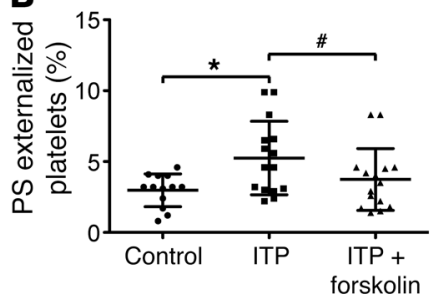

E

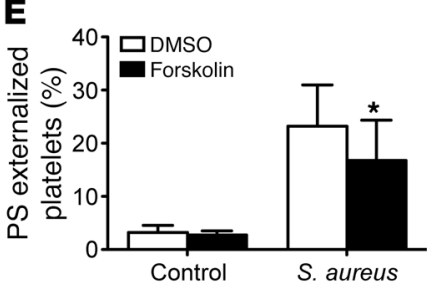

C
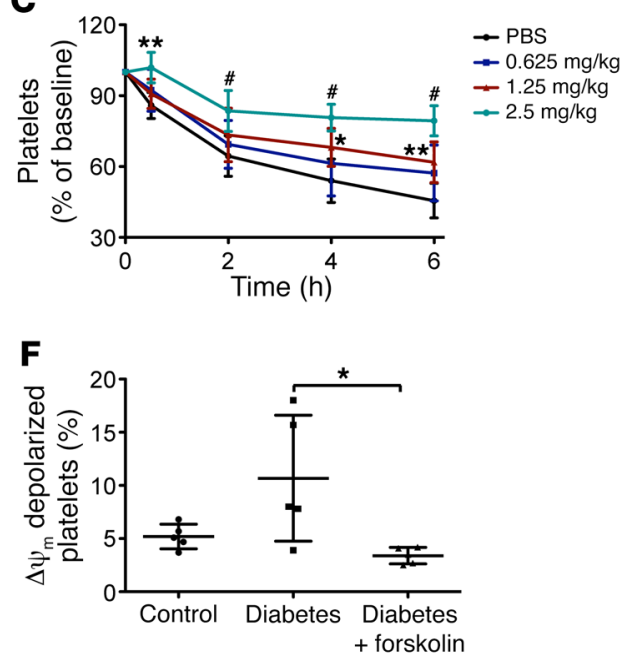

Figure 8. PKA activation protects pathologically stimulated platelets from apoptosis in vitro and clearance in vivo. (A and B) Washed human platelets were incubated with forskolin $(20 \mu \mathrm{M})$ or vehicle at $37^{\circ} \mathrm{C}$ for 30 minutes and further incubated with serum from ITP patients or healthy controls $(1: 1$ volume) for 12 hours. $\Delta \psi_{\mathrm{m}}$ Depolarization (A) and PS exposure (B) are shown. ${ }^{*} P<0.05 ;{ }^{\#} P<0.001$, Mann-Whitney $U$ test for control versus ITP. ${ }^{* *} P<0.01$; ${ }^{\#} P<0.001$, Wilcoxon matched-pairs signed rank test for ITP versus ITP plus forskolin. (C) ICR mice were injected with 8-Br-cAMP or vehicle and further injected with 2.4G2. After 10 minutes, R300 was injected into the mice $(n=6)$. Data are represented as mean of normalized platelet counts (time point 0 : $100 \%) \pm$ SD from 3 independent experiments. ${ }^{*} P<0.05 ;{ }^{* *} P<0.01 ;{ }^{\#} P<0.001$, 2-way ANOVA. (D and E) Human platelets were incubated with forskolin $(5 \mu \mathrm{M})$ or vehicle at $37^{\circ} \mathrm{C}$ for 15 minutes and further incubated with S. aureus (1:20) or vehicle for 90 minutes. Data are represented as mean \pm SD of $\Delta \psi_{\mathrm{m}}$ depolarization (D) and PS exposure (E) from 3 independent experiments. ${ }^{*} P<0.05$; ${ }^{*} P<0.01$, Student's $t$ test. (F) $\Delta \psi_{\mathrm{m}}$ depolarization of platelets incubated with forskolin and serum from diabetes patients or healthy controls. ${ }^{*} P<0.05$, Student's $t$ test.

In addition to apoptosis, there are other kinds of storage lesions (41), contributing to clearance of transfused platelets. We therefore investigated whether prevention of platelet apoptosis by PKA activation could rescue stored platelets from rapid clearance in mice. Similar to the findings with human platelets, PKA inhibitor H89 promoted, but PKA stimulator forskolin reduced, apoptotic events in stored mouse platelets (data not shown). Notably, forskolin obviously protected the stored platelets from clearance in vivo; in contrast, $\mathrm{H} 89$ accelerated the clearance (Figure 7C). Moreover, unlike vehicle-treated platelets, forskolin-treated stored platelets retained the functions of aggregation in vitro (Figure 7, D and E) and thrombosis formation in vivo (Figure 7F). These data demonstrate that PKA activator protects platelets from apoptosis in vitro and prevents transfused platelet clearance in vivo.

PKA activation protects pathologically stimulated platelets from apoptosis in vitro and clearance in vivo. Autoantibody-induced platelet apoptosis results in rapid platelet destruction in ITP patients $(4,42)$. Therefore, incubation of normal platelets with serum from ITP patients that contains autoantibodies induced platelet apoptosis (Figure 8, A and B). However, preincubation of platelets with forskolin markedly reduced apoptotic events in the serum-treated platelets (Figure 8, A and B). To further investigate the effect of the PKA activator on antibody-induced platelet clearance in vivo, we set up a murine model of ITP with R300. The mice were preinjected with Fc receptor blocker to inhibit Fc-dependent platelet destruction. Figure $8 \mathrm{C}$ shows that the PKA activator dosedependently rescued R300-induced platelet destruction. Moreover, PKA activator effectively prevented apoptotic events in platelets incubated with $S$. aureus isolated from sepsis patients (Figure 8, D and E). Forskolin also significantly inhibited $\Delta \Psi_{\mathrm{m}}$ depolariza- tion in platelets incubated with plasma from patients with diabetes (Figure 8F). These data validate that PKA is an upstream regulator of platelet apoptosis induced by different pathophysiological stimuli and, more importantly, suggest novel therapeutic strategies for thrombocytopenia in patients with ITP, sepsis, and diabetes.

\section{Discussion}

In this study, we show that PKA activity is markedly reduced in in vitro-aged platelets or platelets from patients with ITP, sepsis, or diabetes. Inhibition or genetic deletion of PKA incurs intrinsic programmed platelet apoptosis in vitro and rapid platelet clearance in vivo. We demonstrate that PKA regulates platelet apoptosis through mediating BAD Ser155 phosphorylation. Notably, activation of PKA protects platelets from apoptosis induced by storage and pathological stimuli in vitro and elevates peripheral platelets in vivo.

Strikingly, we found that PKA activity was reduced in platelets during storage and in platelets from different patients. PKA activity is mainly regulated by cAMP, the level of which is determined by adenylate cyclase (12) and phosphodiesterases (13) in platelets. Endothelium-derived prostacyclin $\left(\mathrm{PGI}_{2}\right)$ has been determined to be the main physiologic stimulator of cAMP production through activation of adenylate cyclase in platelets, which are marginalized to the periphery of the vessel, facilitating constant exposure to $\mathrm{PGI}_{2}$ released by endothelial cells (Figure 5F) (43). $\mathrm{PGI}_{2}$ exists in plasma with a half-life of only 8.4 to 13.0 minutes, after which it is converted to an inactive stable form (44). Therefore, it is likely that the reduced PKA activity in stored platelets is, at least in part, caused by the absence of $\mathrm{PGI}_{2}$ stimulation. In support of this hypothesis, the addition of PKA activator protected stored platelets from apoptosis (Figure 7, A and B). Our findings suggest that 
trace amounts of thrombin generated in the circulation reduce PKA activity and result in platelet apoptosis in different patients. However, this suggestion does not exclude the possibility that some pathological stimuli induce platelet apoptosis through other mechanisms. For example, platelet apoptosis induced by antiGPIb $\alpha$ antibodies was observed here and reported previously (42). Apoptotic platelets expose PS and release microparticles that incur a procoagulant profile, leading to generation of thrombin. The mutual promoted effects may explain the subtle balance between thrombosis and hemostasis in some diseases (e.g., ITP) and also suggest possible severe consequences once the balance is broken (e.g., disseminated intravascular coagulation).

We demonstrate that PKA regulates platelet apoptosis through regulation of the interaction of BAD with prosurvival BCL-XL. PKA inhibition resulted in dephosphorylation of BAD at Ser155, which released 14-3-3 and sequestered prosurvival BCL-XL on mitochondria, leading to apoptosis. In support of our observation, platelet life span was extended in Bad-KO mice, leading to a modest elevation of peripheral platelets (9). Our finding provides an explanation of the mechanism for this observation (Figure 5F). We further demonstrate that $\mathrm{Bad}$ deficiency is protective against PKA inhibitors-induced platelet apoptosis in vitro (Supplemental Figure 15) and platelet clearance in vivo (Supplemental Figure 17). Moreover, consistent with our observations, the $\mathrm{BH} 3$ mimetic ABT-737, which has a binding profile similar to that of BAD (45), incurred platelet apoptosis in vitro and acute thrombocytopenia in vivo $(6,8)$. We demonstrate that PKA activator protect platelets from apoptosis induced by ABT-737 (Supplemental Figure 19).

BCL-XL has been found to play a key role in regulating platelet apoptosis (6). Degradation of BCL-XL in aged platelets was presumed to initiate apoptosis and determine platelet life span (6). Consistent with previous reports, we observed BCL-XL degradation in stored platelets. However, PKA activator prevented not only apoptosis in the stored platelets (Figure 7), but also BCL-XL degradation in the platelets (Supplemental Figure 20). In addition, no obvious BCL-XL degradation was observed in PKA inhibitorinduced acute platelet apoptosis (Figure 5). Apoptosis per se incurs proteolysis. Thus, our findings suggest that the decline of BCL$\mathrm{XL}$ in apoptotic platelets might be a consequence rather than an initiator of apoptosis. Importantly, activation of PKA prevented apoptosis in aged platelets and protected platelets from apoptosis induced by pathological stimuli as well, suggesting that apoptosis initiated by $\Delta \Psi_{\mathrm{m}}$ depolarization is an irreversible process. Thus, these findings support PKA as an upstream regulator for initiating and regulating platelet apoptosis.

Apoptosis contributes to storage lesion, which severely limits platelet shelf life $(6,8,21)$. Several groups have tried to reduce storage lesion by inhibiting apoptosis-related enzymes such as caspases. However, although the enzymes were inhibited, the alleviation for storage lesion was not significant. It is noteworthy that, despite the mechanism remaining unclear, inhibition of p38 MAPK $(46)$ or p38 MAPK-mediated GPIb $\alpha$ shedding $(46,47)$ significantly improves the quality and posttransfusion survival and function of stored platelets. We and others previously reported that GPIb $\alpha$-dependent signaling could induce platelet apoptosis and that association of 14-3-3 with the cytoplasmic domain of GPIb $\alpha$ is essential for apoptotic signaling $(7,48,49)$. Storage incurs acti- vation of p38 MAPK and p38 MAPK-mediated cytosolic phospholipase A2, resulting in release of arachidonic acid from membrane phospholipids, which transfers 14-3-3 to the cytoplasmic domain of GPIb $\alpha(48,49)$. In the meantime, p38 MAPK activates ADAM17, leading to GPIb $\alpha$ ectodomain shedding (47), which enhances GPIb $\alpha$ clustering and association of 14-3-3 with the cytoplasmic domain of GPIb $\alpha$ (50). 14-3-3 competes with BCL-XL to bind BAD, thus increasing 14-3-3-GP1ba interaction $(38,39)$. Thus, elevation of 14-3-3-GPIb $\alpha$ interaction should enhance sequestration of prosurvival BCL-XL by BAD on mitochondria, leading to platelet apoptosis. This hypothesis is supported by observations made in platelets rewarmed after cold storage, in which enhancement of 14-3-3-GPIb $\alpha$ interaction reduced association of 14-3-3 with BAD, resulting in platelet apoptosis $(50,51)$. Therefore, the mechanism by which inhibition of p38 MAPK or GPIb $\alpha$ shedding extends platelet shelf life is likely through the inhibition of 14-3-3-BCL$\mathrm{XL}-\mathrm{BAD}$-mediated platelet apoptosis. In light of current findings, elevation of PKA activity, similar to the inhibition of p38 MAPK or GPIb $\alpha$ shedding, may represent a strategy for preventing apoptosis and extending the shelf life of platelets.

Thrombocytopenia, which occurs with many diseases or during treatments, leads to life-threatening hemorrhage worldwide every day. However, the pathogenesis of thrombocytopenia is not yet fully understood, leading to difficulty in selecting appropriate treatment. For example, there are many refractory ITP patients who do not respond to conventional treatments, and the reason for this remains unclear (52). Similarly, little is known about the pathogenesis of thrombocytopenia during bacterial infections or diabetes $(2,3,5)$. According to current observations, once thrombin is generated in the circulation, it should reduce PKA activity in platelets, leading to apoptosis and destruction. Thus, our finding appears to disclose a common pathogenesis of thrombocytopenia that occurred in all of the thrombin-generated diseases, including malignant, metabolic, and cardiovascular diseases. Furthermore, we found that PKA activation effectively protects platelets from apoptosis provoked by different stimulations and elevates peripheral platelets. Therefore, these findings suggest a profound therapeutic strategy for thrombocytopenia induced by different pathological stimuli. On the other hand, as PKA inhibition incurs platelet apoptosis and rapid clearance in vivo, targeting PKA appears to be a promising strategy for treating platelet-excessive or hyperactive diseases such as essential thrombocytosis and thrombosis.

In conclusion, our study identifies PKA as a homeostatic regulator of platelet apoptosis to determine platelet life span and survival. Regulation of PKA activity represents a strategy for extending platelet shelf life and has profound implications for the treatment of platelet number-related diseases.

\section{Methods}

Patients and healthy volunteers. Twenty-five subjects with ITP (Supplemental Table 1), 11 subjects with diabetes (Supplemental Table 2), 15 subjects with sepsis (Supplemental Table 3), and age- and sexmatched healthy control subjects were recruited for the studies.

Mice. PKA C $\alpha$ conditional KO mice were generated by introducing a loxP site on either side of exons 6, 7, and 8 of the Pkaca gene (Figure 4A). For each targeting site, gRNAs were designed. gRNAs and Cas 9 
mRNAs were transcribed by T7 RNA polymerase. The Pkaca-targeting gRNAs, Cas9 mRNAs, and donor DNAs with loxP-flanked targeted exons were microinjected into the 1-cell embryos to generate chimera. Targeted Pkaca floxed founder mice were identified by PCR and sequencing, and then these founder mice were bred to C57BL/6J mice to verify the germline transmission by PCR and sequencing (Supplemental Figure 11). Mice with KO specific to megakaryocytes and platelets were produced by crossing the floxed mice with transgenic mice expressing Pf4 promoter-driven Cre recombinase (008535, Pf4-Cre; The Jackson Laboratory). All of the KO processes were produced on a C57BL/6J background. Genotyping was performed by PCR, and Pkaca deficiency was verified by immunoblotting. $\mathrm{Bad}^{-/-}$mice were generated on a 129/SvJ background as described previously (53) and had been backcrossed to a C57BL/6 background for more than 10 generations. C57BL/6 WT mice and ICR WT mice were purchased from JOINN Laboratories. Male mice were analyzed at 6 to 12 weeks of age unless otherwise specified.

Antibodies and reagents. Antibodies against $\beta$-actin (catalog 4979), mouse PKA C- $\alpha$ (catalog 4782), p53 (catalog 2524), phospho-p53 (ser15) (catalog 9284), and human PKA C- $\alpha$ (catalog 5842) were from Cell Signaling Technology. Antibodies against BAD (ab32445), phospho-BAD (Ser155) (ab28825), CD16+CD32 (2.4G2) (ab210219), BIM (ab7888) and BCL-2 (ab692), anti-mouse IgG H\&L (Alexa Fluor 555) (ab150114), anti-rabbit IgG H\&L (Alexa Fluor 488) (ab150077), FITC-conjugated anti-CD41 antibody (ab19708), and ThrombinAntithrombin Complex Human ELISA Kit (ab108907) were from Abcam. Anti-phospho-GPIb $\beta$ (Ser166) and anti-GPIb $\beta$ antibodies were gifts from Xiaoping $\mathrm{Du}$ (University of Illinois at Chicago, Chicago, Illinois, USA) (20). $\mathrm{N}$-[2-((p-Bromo Cinnamyl) amino)ethyl]-5-isoquinoline sulfonamide (H89) (S1644), cyclosporin A (S1563), JC-1 (C2005), caspase-3 activity assay kit (C1115), and forskolin (S1612) were purchased from Beyotime Biotechnology. A mixture of purified rat monoclonal antibodies against mouse GPIba (R300) was from Emfret Analytics. Lactadherin (FFO805) was from Haematologic Technologies. Glutaraldehyde solution (G5882), thiazole orange (390062), 8-Br-cAMP (B7880), Rp-cAMPS (A165), ristocetin, DMSO (V900090), N-hydroxysuccinimido-biotin (NHS-biotin), gelsolin antibody (G4896), and $\beta$-actin antibody (A5316) were purchased from Sigma-Aldrich. Mitochondria Isolation Kit (catalog 89874) was from Thermo Fisher Scientific. Calcein-AM was purchased from Dojindo Laboratories. Annexin V-FITC Kit (LHK601-100) was from Jiamay Biotechnology. $\alpha$-Tubulin antibody (catalog 7597) was from ProSci. PE-anti-mouse CD41 antibody (catalog 133906) and FITC-conjugated mouse anti-human P-selectin antibody (catalog 304904) were from BioLegend. Antibodies against caspase-3 (sc-271028), phosphoBAD Ser155 (sc-133356), BCL-XL (sc-8392), BAK (sc-832) and BAX (sc-493), A23187 (sc-3591), Protein G Plus-agarose (sc-2002), Protein A/G Plus-agarose (sc-2003), mouse IgG (sc-2025), normal rabbit IgG (sc-2026), HRP-conjugated goat anti-rabbit IgG (sc-2004), and HRP-conjugated goat anti-mouse IgG (sc-2005) were purchased from Santa Cruz Biotechnology Inc. Thrombin, collagen, ADP, and Chrono-Lume were from Chronolog. FITC-conjugated PAC-1 (catalog 340507) was from BD Bioscience.

PCR genotyping. To genotype the weaned pups, genomic DNA was extracted from about $0.5 \mathrm{~cm}$ of mouse tail and analyzed by PCR and sequencing. The mice containing the floxed allele were identified by PCR and sequencing analysis using primers flanking the $5^{\prime}$ loxP site and $3^{\prime}$
loxP site. The sense loxp1-F1 (5'-ATGTCCTACTATGTCAGGCTCC-3') and antisense loxp1-R2 (5'-TCAAATGTAGACTAAAGGCTGGG-3') flanking the $5^{\prime}$ loxP site amplify a 158-bp band from the WT allele and a 192-bp band from the floxed allele. The sense loxp2-F1 (5'-ACGGATTTCAGCTCTGGAAGGA-3') and antisense loxp2-R1 (5'-TTAGTTGAGCCATCAACCCCTGTC-3') flanking the 3' loxP site amplify a 216bp band from the WT allele and a 250-bp band from the floxed allele. The sense PKA C $\alpha-F$ (5'-GACACAGGGTCTCACTTTGTAG- $3^{\prime}$ ) and antisense PKA C $\alpha$-R (5'-TGATGTGGCGCAGCTTCCTGAC-3') flanking the $5^{\prime}$ and $3^{\prime}$ loxP sites were used to identify the correctness of both the $5^{\prime}$ loxP and $3^{\prime}$ loxP sequences. Mice containing the Cre allele were determined by the following primers: Cre sense: $5^{\prime}$-CCCATACAGCACACCTTTTG-3' and antisense: 5'-TGCACAGTCAGCAGGTT-3'; 450 bp band for Cre allele, no band for WT allele. PCR was performed as follows: briefly, in a $25 \mu \mathrm{l}$ reaction volume, 100 ng of genomic DNA and 10 pmol of each primer (loxP1-F1, loxP1-R2, loxP2-F1, loxP2-R1, PKA C $\alpha-F$, and PKA $\mathrm{C} \alpha-\mathrm{R}$ ) were amplified in PCR buffer supplemented with 0.5 units of Taq polymerase (MightyAmp Takara). The cycling parameters were as follows: $98^{\circ} \mathrm{C}$ for 5 minutes; 32 cycles of 10 seconds at $98^{\circ} \mathrm{C}, 20$ seconds at $60^{\circ} \mathrm{C}$, and 1 minute at $68^{\circ} \mathrm{C}$; and a final extension for $5 \mathrm{~min}-$ utes at $68^{\circ} \mathrm{C} .10 \mu \mathrm{l}$ of each reaction mixture was separated on a $1.0 \%$ agarose gel in $1 \times$ Tris acetate-EDTA buffer.

Bacterial culture and preparation. S. aureus (ATCC 29213), Klebsiella pneumoniae (ATCC 700603), E. coli DH5a, Pseudomonas aeruginosa (ATCC 27853), Enterococcus faecalis (ATCC 29212), and Staphylococcus haemolyticus (BD 3569) were obtained from the Clinical Laboratory of the Second Affiliated Hospital of Soochow University. All strains from frozen stocks were cultured in Luria-Bertani (LB) at $37^{\circ} \mathrm{C}$ for 15 hours. Cells were centrifuged at 2,000 g for 5 minutes and resuspended in fresh LB media with $30 \%$ glycerin and stored at $-80^{\circ} \mathrm{C}$ before use. The amounts of bacteria were determined by a petri dish colony-counting method, and the corresponding concentrations of each bacterium were as follows: $S$. aureus, $1.65 \times 10^{10} \mathrm{CFU} / \mathrm{ml}$; K. pneumoniae, $1.67 \times$ $10^{10} \mathrm{CFU} / \mathrm{ml}$; E. coli, $1.71 \times 10^{10} \mathrm{CFU} / \mathrm{ml}$; P. aeruginosa, $1.14 \times 10^{11} \mathrm{CFU} /$ $\mathrm{ml}$; E. faecalis, $2.00 \times 10^{10} \mathrm{CFU} / \mathrm{ml}$; and S. haemolyticus, $1.80 \times 10^{11}$ $\mathrm{CFU} / \mathrm{ml}$. Just before experiments, bacteria were centrifuged at 2,000 $g$ for 5 minutes and resuspended in modified Tyrode's buffer (MTB) (12.1 mM NaHCO$, 136.9 \mathrm{mM} \mathrm{NaCl}, 5.6 \mathrm{mM}$ D-glucose, $2.6 \mathrm{mM} \mathrm{KCl}$, $2.4 \mathrm{mM}$ HEPES, $1 \mathrm{mM} \mathrm{CaCl}_{2}, 1 \mathrm{mM} \mathrm{MgCl}_{2}$, and 0.1\% BSA, $\mathrm{pH}$ 7.4).

Platelet counts and preparation. Platelet and blood cell counts were performed with Sysmex XP-100 Hematologic Analyzer (Sysmex Corporation). The platelets from healthy volunteers were prepared as previously described (7). Briefly, whole blood was drawn from the inferior vena cava and anticoagulated with $1 / 7$ volume of acid-citrate-dextrose (ACD) (2.5\% trisodium citrate, $2.0 \% \mathrm{D}$-glucose, $1.5 \%$ citric acid). PRP was collected from whole blood by $200 \mathrm{~g}$ centrifugation for 11 minutes. Platelets were washed twice with CGS buffer $(0.123 \mathrm{M} \mathrm{NaCl}, 0.033 \mathrm{M}$ D-glucose, $0.013 \mathrm{M}$ trisodium citrate, $\mathrm{pH}$ 6.5), and resuspended in MTB to a final concentration of $3 \times 10^{8} / \mathrm{ml}$. Then washed platelets were incubated at room temperature (RT) for 2 hours before use. For the preparation of mouse platelets, whole blood from mice was collected from the eye socket vein using $1 / 7$ volume of ACD as anticoagulant. Platelets were washed with CGS buffer and resuspended in MTB to a concentration of $3 \times 10^{8} / \mathrm{ml}$ and allowed to incubate at $22^{\circ} \mathrm{C}$ for 1 to 2 hours. For the preparation of platelets from patients, whole blood was drawn from the inferior vena cava and anticoagulated with ACD. PRP was collected from the whole blood. PRP was centrifugated 
at $200 \mathrm{~g}$ for 2 minutes to separate plasma and platelets. Platelets were resuspended in MTB to a final concentration of $3 \times 10^{8} / \mathrm{ml}$.

In vivo platelet experiments. C57BL/ 6 mice (6 to 8 weeks) or male ICR mice (4 to 5 weeks) were injected with different concentrations of PKA inhibitor (Rp-cAMPS), activator (8-Br-cAMP), or vehicle control (PBS) through the tail vein. In experiments with the mouse ITP model, male ICR mice ( 4 to 5 weeks) were intravenously injected with a single dose of PKA activator 8-Br-cAMP $(0.625,1.25$, or $2.5 \mathrm{mg} / \mathrm{kg})$ with or without intraperitoneal injection of anti-mouse CD16/CD32 antibody $(2.4 \mathrm{G} 2,0.6 \mathrm{mg} / \mathrm{kg})(\mathrm{BD})$ to block Fc-dependent clearance. After 10 minutes, the mice were intraperitoneally injected with anti-platelet antibody (R300, $0.1 \mathrm{mg} / \mathrm{kg}$ ) or vehicle control. Whole blood was collected from eye socket veins at certain time points, and platelet count was performed with Sysmex XP-100 Hematologic Analyzer.

Platelet clearance. Washed mouse platelets in MTB $\left(3 \times 10^{8} / \mathrm{ml}\right)$ were incubated with $\mathrm{H} 89(25 \mu \mathrm{M})$, forskolin $(5 \mu \mathrm{M})$, or vehicle at $22^{\circ} \mathrm{C}$ for 72 hours. The platelets were labeled with $5 \mu \mathrm{M}$ of calcein and injected intravenously into C57BL/6 WT mice through the postglomus venous plexus. Whole blood was collected from eye socket veins of the mice at 1 (baseline), 15, and 30 minutes after injection, and the labeled platelets were analyzed with flow cytometry (FC 500, Beckman-Coulter).

Reticulated platelet counts. Fresh blood from mice was collected from eye socket veins using $1 / 7$ volume of ACD as an anticoagulant and was incubated with $0.5 \mu \mathrm{g} / \mathrm{ml}$ thiazole orange (Sigma-Aldrich) and $20 \mu \mathrm{g} / \mathrm{ml} \mathrm{PE}$-anti-mouse CD41 antibody (BioLegend). The sample was incubated at RT in the dark for 15 minutes. Reticulated platelets were analyzed by flow cytometer (FC 500, Beckman-Coulter).

ELISA for PKA activity. PKA activity was examined by ELISA with a PKA activity kit (catalog ADI-EKS-390A; Enzo Life Science Inc.). Briefly, platelet lysate $(30 \mu \mathrm{l})$ was added into each well of a microtiter plate, followed by adding $10 \mu \mathrm{l}$ diluted ATP. The wells were incubated at $30^{\circ} \mathrm{C}$ for 90 minutes and then were emptied and $40 \mu \mathrm{l}$ of phosphospecific substrate antibody was added. After 60 minutes, the wells were washed and $40 \mu$ of HRP-conjugated anti-rabbit IgG was added. After 30 minutes, the wells were washed and $60 \mu \mathrm{TMB}$ substrate was added. The reaction was stopped after 30 to 60 minutes. The plates were read at $450 \mathrm{~nm}$ with a Variskan Flash Spectral Scanning Multimode Reader (Thermo Scientific). The relative PKA kinase activities were calculated by the following equation: (sample average absorbance - blank average absorbance)/quantity of crude protein used per assay.

ELISA for thrombin generation. Thrombin-antithrombin (TAT) complexes formed following the neutralization of thrombin by antithrombin III have been used as a surrogate marker for thrombin generation. TAT complexes were examined by ELISA with the ThrombinAntithrombin Complex Human ELISA Kit. Briefly, plasma of patients (ITP, sepsis, and diabetes) was added into each well of a microtiter plate and incubated for 2 hours. Each well was washed and $50 \mu \mathrm{l}$ of $1 \times$ Biotinylated Thrombin-Antithrombin Complex antibody was added, followed by incubation for 1 hour. Then the well was washed and $50 \mu \mathrm{l}$ of $1 \times$ SP conjugate was added. After 30 minutes, the well was washed and $50 \mu \mathrm{l}$ of chromogen substrate was added. The reaction was stopped after 30 minutes. The plates were read at $450 \mathrm{~nm}$ with a Variskan Flash Spectral Scanning Multimode Reader (Thermo Scientific).

Mitochondrial $\Delta \psi_{m}$ depolarization assay. PRP or washed platelets in MTB $\left(3 \times 10^{8} / \mathrm{ml}\right)$ were incubated with or without PKA inhibitors or activators, thrombin, or vehicle control at different temperatures for different times. The lipophilic cationic probe JC-1 was added to the pretreated platelets to a final concentration of $2 \mu \mathrm{g} / \mathrm{ml}$ and incubated at $37^{\circ} \mathrm{C}$ in the dark for 5 minutes. The treated samples were detected by flow cytometry. Depolarization is characterized as the decrease in the content of JC-1 aggregates, as reflected in the decrease of FL2 fluorescence.

PS externalization assay. The pretreated platelets were mixed with annexin V-binding buffer and annexin V-FITC at a 10:50:1 ratio. Samples were gently mixed and incubated at RT for 15 minutes in the dark, then analyzed by flow cytometry. In some experiments, treated platelets were incubated with lactadherin to a final concentration of $5 \mu \mathrm{g} /$ $\mathrm{ml}$ in the dark for 30 minutes, then analyzed by flow cytometry.

Caspase- 3 activity assay. Platelets $\left(3 \times 10^{8} / \mathrm{ml}\right)$ were incubated with $\mathrm{H} 89(25 \mu \mathrm{M})$ or DMSO at $22^{\circ} \mathrm{C}$ for 30 minutes. The caspase- 3 activity assay was performed on 96-well microtiter plates by adding $10 \mu \mathrm{l}$ of platelet lysate per sample in $80 \mu \mathrm{l}$ reaction buffer and $10 \mu \mathrm{l}$ caspase-3 substrate (Ac-DEVD-pNA, $2 \mathrm{mM}$ ). Samples were further incubated at $37^{\circ} \mathrm{C}$ for 4 hours and were determined by an ELISA reader at an absorbance of $405 \mathrm{~nm}$. The specific activity of caspase-3, normalized for the total protein of the sample, was then indicated as fold of the baseline caspase-3 activity of the vehicle control-treated platelets.

Platelet shrinkage. Washed platelets were incubated with indicated concentrations of $\mathrm{H} 89$, vehicle control, or A23187 (positive control) at $22^{\circ} \mathrm{C}$ for 30 minutes. FITC-conjugated anti-CD 41 antibody was mixed with pretreated platelets at a 1:10 ratio. Samples were gently mixed and incubated at RT for 10 minutes in the dark. Then platelets were fixed with $1 \%$ cold paraformaldehyde, further incubated at RT in the dark for 30 minutes, and analyzed by flow cytometry. After acquisition, FSC-FL1 dot plots were analyzed. Platelet shrinkage was determined by analyzing FSC dot plots as the decrease in FSC characteristics of CD41-positive events and expressed as the mean FSC. A23187 and DMSO were set as positive and negative controls, respectively.

Scanning electron microscopy of platelet morphology. Washed platelets $\left(3 \times 10^{8} / \mathrm{ml}\right)$ were incubated with $\mathrm{H} 89$ or vehicle control (DMSO) at $37^{\circ} \mathrm{C}$ for 160 minutes. Then platelets were fixed at 30 minutes with $2.5 \%$ glutaraldehyde. Fixed platelets were gradually dehydrated in alcohol and acetonitrile. Specimens were taken by acetonitrile freezevacuum-dry technique and glued on the sample table, then coated with gold in a vacuum sputter coater and scanned with a Philips XL-20 scanning electron microscope, whose accelerated voltage was $15.0 \mathrm{KV}$.

Isolated mitochondria from platelets. The mitochondria of platelets were isolated using the Mitochondria Isolation Kit (Thermo Fisher Scientific). Briefly, washed human platelets $\left(3 \times 10^{8} / \mathrm{ml}\right)$ were incubated with H89 $(25 \mu \mathrm{M})$ or forskolin $(10 \mu \mathrm{M})$ at RT for 120 minutes. Platelets were then suspended in mitochondrion isolation buffer A $(400 \mu \mathrm{l})$ for 2 minutes, buffer B ( $5 \mu \mathrm{l})$ for 5 minutes, and buffer C (400 $\mu$ l) for 2 minutes, followed by centrifugation. The supernatant was centrifuged at $12,000 \mathrm{~g}$ for 10 minutes. The pellet containing the mitochondria was washed with buffer $\mathrm{C}$. The isolated mitochondria were verified and analyzed by Western blotting or confocal microscopy.

Confocal microscopy. Isolated mitochondria from platelets were fixed with $4 \%$ paraformaldehyde in PBS for 10 minutes and then permeabilized with $0.5 \%$ Triton-X 100 in PBS with 3\% BSA for 10 minutes. The mitochondria were incubated with different antibodies, including rabbit monoclonal anti-BAD antibody (Abcam, ab32445) and mouse anti-BCL-XL antibody (Santa Cruz Biotechnology Inc., sc-8392) at 1:100 dilution in PBS with $10 \%$ BSA at $4^{\circ} \mathrm{C}$ overnight. The mitochondria were then washed and incubated with Alexa Fluor 555conjugated anti-mouse IgG H\&L (Abcam, ab150114) or Alexa Fluor 
488-conjugated anti-rabbit IgG H\&L (Abcam, ab150077). The stained mitochondria were observed with a LEICA TCS SP8 confocal microscope with a $\times 63$ oil immersion lens.

Western blots. Washed platelets $\left(3 \times 10^{8} / \mathrm{ml}\right)$ were incubated with various concentrations of PKA activators, inhibitors, or vehicle control at $37^{\circ} \mathrm{C}$ or RT for indicated times and lysed with an equal volume of lysis buffer on ice for 30 minutes. Proteins were separated by SDSPAGE. After blocking, membranes were incubated with different primary antibodies and protein bands were visualized by the ECL Chemiluminescence System on Kodak film. Quantification was performed with ImageJ software (NIH).

Coimmunoprecipitation. Washed platelets $\left(3 \times 10^{8} / \mathrm{ml}\right)$ were preincubated with PKA activators, inhibitors, or vehicle control at $37^{\circ} \mathrm{C}$ or RT for indicated times and then were lysed with equal volumes of $2 \times \mathrm{NP} 40$ lysis buffer (100 mM Tris, pH 7.4, 2\% NP40, $20 \mathrm{mM} \mathrm{MgCl}_{2}, 300 \mathrm{mM}$ $\mathrm{NaCl}, 2 \mathrm{mM}$ PMSF, $2 \mathrm{mM} \mathrm{NaF}$, and $2 \mathrm{mM} \mathrm{Na}_{3} \mathrm{VO}_{4}$ ) containing protease inhibitor cocktail tablets on ice for 30 minutes. After centrifugation at $17,000 \mathrm{~g}$ and $4^{\circ} \mathrm{C}$ for 10 minutes, the supernatants were immunoprecipitated with different antibodies overnight. After incubation with Protein A/G PLUS-Agarose beads at $4^{\circ} \mathrm{C}$ for 2 hours (Santa Cruz Biotechnology Inc.), the beads were analyzed by immunoblotting.

Platelet life span analysis. Mice were intravenously injected with $600 \mu \mathrm{g}$ NHS-biotin in buffer containing $140 \mathrm{mM} \mathrm{NaCl}$ and $10 \% \mathrm{DMSO}$. Mice were anesthetized with $2.5 \%$ tribromoethanol $(0.15 \mathrm{ml} / 10 \mathrm{~g})$, and whole blood was collected by capillary tube from the retroorbital venous plexus at various time points and mixed with ACD. Whole blood $(5 \mu \mathrm{l})$ was diluted with MTB $(45 \mu \mathrm{l})$ and stained by PE anti-mouse CD41 antibody $(1 \mu \mathrm{l})$ and FITC-streptavidin $(2.5 \mu \mathrm{l})$ at RT for 1 hour. The percentage of biotinylated platelets was determined by flow cytometry.

Platelet aggregation. Platelet aggregation was recorded in a CHRONO-LOG lumi-aggregometer. PRP or washed platelets $\left(3 \times 10^{8} / \mathrm{ml}\right)$ were incubated with different concentrations of forskolin or vehicle (DMSO) at $22^{\circ} \mathrm{C}$ for 30 minutes or 72 hours. The pretreated PRP or washed platelets were stimulated with different agonists. Platelet aggregation was monitored continuously over 5 to 10 minutes.

Platelet activation. Platelet activation was detected by P-selectin (CD62-P) surface exposure and integrin $\alpha \operatorname{IIb} \beta_{3}$ activation. Washed platelets in MTB $\left(3 \times 10^{8} / \mathrm{ml}\right)$ were incubated with or without thrombin or vehicle control at $37^{\circ} \mathrm{C}$ for 30 minutes. The treated platelets were incubated with FITC-labeled anti-CD62-P antibody or FITClabeled PAC-1 at RT for 20 minutes in the dark and then subjected to flow cytometry analysis.

In vivo thrombosis. The ferric chloride-induced ( $\mathrm{FeCl}_{3}$-induced) mesenteric arteriole thrombosis model was used. Briefly, washed mouse platelets were labeled with calcein-AM $(5 \mu \mathrm{g} / \mathrm{ml})$ and incubated with H89 $(25 \mu \mathrm{M})$, forskolin $(5 \mu \mathrm{M})$, or vehicle (DMSO) at $22^{\circ} \mathrm{C}$ for 30 minutes or 72 hours. The recipient male mice were anesthetized, and the mesentery vascular bed was exteriorized. One arteriole was chosen and visualized with an inverted fluorescent microscope (Leica Microsystems) and recorded on videotape. The mice were inject- ed intravenously with pretreated platelets $\left(5 \times 10^{6} / \mathrm{g}\right)$. Thrombus formation was induced by topical application of $3 \mathrm{~mm}^{2}$ of filter paper soaked with $5 \% \mathrm{FeCl}_{3}$.

Statistics. All data are expressed as mean \pm SD. Numeric data were analyzed using 1-way (for single variant) or 2-way (for multiple variants) ANOVA followed by Bonferroni's adjustment for multiple comparisons. Two groups were compared using 2-tailed Student's $t$ test. The significance of data was assessed using GraphPad Prism 5 software. Differences were considered as significant at $P<0.05$. All experiments requiring the use of animals were subject to randomization based on litter. No animals or samples were excluded from the study. Sample size was predetermined based on the variability observed in prior experiments and on preliminary data. Investigators were not blinded to outcome assessment.

Study approval. All animal experiments were approved by the Ethics Committee of The First Affiliated Hospital of Soochow University. Approval to obtain whole blood samples from healthy volunteers and patients was obtained from the Ethics Committee of the First Affiliated Hospital of Soochow University, and written informed consent was obtained from all subjects according to the Declaration of Helsinki.

\section{Author contributions}

Lili Zhao, JL, CH, and RY designed and performed research and collected, analyzed, and interpreted results. KZ, QC, XM, XL, YZ (9th author), MC, YL, and YN performed animal experiments. JL, YZ (9th author), RH, LC, Ling Zhou, Yunxiao Zhao, and WX performed experimental analysis for patients. Lili Zhao, RY, QC, YZ (11th author), JT, and XM performed mechanistic experiments of apoptosis. CH, Lian Zhao, JL, RH, JT, and MC performed bacterial experiments; AL provided $\mathrm{Bad}^{-/-}$mice; $\mathrm{CR}$ analyzed data. $\mathrm{KD}$ initiated and supervised the project, designed research, analyzed and interpreted results, and wrote the paper.

\section{Acknowledgments}

This work was supported by grants from the Key Program of the National Natural Science Foundation of China (81130008 to KD), the National Natural Science Foundation of China (81570102 to KD and 81500093 to Lili Zhao), the National Key Basic Research Program of China (2012CB526600 to KD), the NIH (R35GM122457), the Priority Academic Program Development of Jiangsu Higher Education Institutions (PAPD), the Jiangsu Provincial Special Program of Medical Science (BL2012005), the Jiangsu Province's Key Medical Center (ZX201102), and the Jiangsu Province's Outstanding Medical Academic Leader Program (to KD).

Address correspondence to: Kesheng Dai, Jiangsu Institute of Hematology, The First Affiliated Hospital and Collaborative Innovation Center of Hematology, Soochow University, Key Laboratory of Thrombosis and Hemostasis, Ministry of Health, Suzhou 215006, China. Phone: 86.512.67781370; Email:kdai@suda.edu.cn.
1. Leeksma CH, Cohen JA. Determination of the life of human blood platelets using labelled diisopropylfluorophosphanate. Nature. 1955;175(4456):552-553.

2. Yeh JJ, Tsai S, Wu DC, Wu JY, Liu TC, Chen A. P-selectin-dependent platelet aggregation and apoptosis may explain the decrease in platelet count during Helicobacter pylori infection. Blood. 2010;115(21):4247-4253.

3. Gafter-Gvili A, et al. Thrombocytopenia in Staphylococcus aureus bacteremia: risk factors and prognostic importance. Mayo Clin Proc.
2011;86(5):389-396.

4. Goette NP, et al. Platelet apoptosis in adult immune thrombocytopenia: insights into the mechanism of damage triggered by autoantibodies. PLoS One. 2016;11(8):e0160563.

5. Tang WH, et al. Aldose reductase-mediated 
phosphorylation of $\mathrm{p} 53$ leads to mitochondrial dysfunction and damage in diabetic platelets. Circulation. 2014;129(15):1598-1609.

6. Mason KD, et al. Programmed anuclear cell death delimits platelet life span. Cell. 2007;128(6):1173-1186.

7. Li S, et al. The glycoprotein Ibalpha-von Willebrand factor interaction induces platelet apoptosis. J Thromb Haemost. 2010;8(2):341-350.

8. Kodama T, et al. BH3-only activator proteins Bid and Bim are dispensable for Bak/Bax-dependent thrombocyte apoptosis induced by Bcl-xL deficiency: molecular requisites for the mitochondrial pathway to apoptosis in platelets. J Biol Chem. 2011;286(16):13905-13913.

9. Kelly PN, et al. Individual and overlapping roles of $\mathrm{BH} 3$-only proteins Bim and Bad in apoptosis of lymphocytes and platelets and in suppression of thymic lymphoma development. Cell Death Differ. 2010;17(10):1655-1664.

10. Niswender CM, Ishihara RW, Judge LM, Zhang C, Shokat KM, McKnight GS. Protein engineering of protein kinase A catalytic subunits results in the acquisition of novel inhibitor sensitivity. J Biol Chem. 2002;277(32):28916-28922.

11. Skålhegg BS, Huang Y, Su T, Idzerda RL, McKnight GS, Burton KA. Mutation of the Calpha subunit of PKA leads to growth retardation and sperm dysfunction. Mol Endocrinol. 2002;16(3):630-639.

12. Hanoune J, Defer N. Regulation and role of adenylyl cyclase isoforms. Annu Rev Pharmacol Toxicol. 2001;41:145-174.

13. Omori K, Kotera J. Overview of PDEs and their regulation. Circ Res. 2007;100(3):309-327.

14. Insel PA, Zhang L, Murray F, Yokouchi H, Zambon AC. Cyclic AMP is both a pro-apoptotic and anti-apoptotic second messenger. Acta Physiol (Oxf). 2012;204(2):277-287.

15. Keshwani MM, et al. Mechanisms of cyclic AMP/protein kinase A- and glucocorticoidmediated apoptosis using S49 lymphoma cells as a model system. Proc Natl Acad Sci U S A. 2015;112(41):12681-12686.

16. Beck F, et al. Time-resolved characterization of cAMP/PKA-dependent signaling reveals that platelet inhibition is a concerted process involving multiple signaling pathways. Blood. 2014;123(5):e1-e10.

17. Raslan Z, Naseem KM. The control of blood platelets by cAMP signalling. Biochem Soc Trans. 2014;42(2):289-294.

18. Bergmeier W, et al. Metalloproteinase inhibitors improve the recovery and hemostatic function of in vitro-aged or -injured mouse platelets. Blood. 2003;102(12):4229-4235.

19. Pereira J, et al. Platelet aging in vivo is associated with activation of apoptotic pathways: studies in a model of suppressed thrombopoiesis in dogs. Thromb Haemost. 2002;87(5):905-909.

20. Bodnar RJ, Xi X, Li Z, Berndt MC, Du X. Regulation of glycoprotein Ib-IX-von Willebrand factor interaction by cAMP-dependent protein kinase-mediated phosphorylation at Ser 166 of glycoprotein Ib(beta). J Biol Chem. 2002;277(49):47080-47087.

21. Jackson SP, Schoenwaelder SM. Procoagulant platelets: are they necrotic? Blood. 2010;116(12):2011-2018.

22. Petros S, Kliem P, Siegemund T, Siegemund R. Thrombin generation in severe sepsis. Thromb Res. 2012;129(6):797-800.

23. Tripodi A, et al. Hypercoagulability in patients with type 2 diabetes mellitus detected by a thrombin generation assay. J Thromb Thrombolysis. 2011;31(2):165-172.

24. Álvarez-Román MT, et al. Procoagulant profile in patients with immune thrombocytopenia. $\mathrm{Br} J$ Haematol. 2016;175(5):925-934.

25. Jantzen HM, Milstone DS, Gousset L, Conley PB, Mortensen RM. Impaired activation of murine platelets lacking $\mathrm{G}$ alpha(i2). J Clin Invest. 2001;108(3):477-483.

26. Zhang W, Colman RW. Thrombin regulates intracellular cyclic AMP concentration in human platelets through phosphorylation/activation of phosphodiesterase 3A. Blood.2007;110(5):1475-1482.

27. Kroemer G, Reed JC. Mitochondrial control of cell death. Nat Med. 2000;6(5):513-519.

28. Leytin V, Allen DJ, Mutlu A, Gyulkhandanyan AV, Mykhaylov S, Freedman J. Mitochondrial control of platelet apoptosis: effect of cyclosporin A, an inhibitor of the mitochondrial permeability transition pore. Lab Invest. 2009;89(4):374-384.

29. Schoenwaelder SM, et al. Two distinct pathways regulate platelet phosphatidylserine exposure and procoagulant function. Blood. 2009;114(3):663-666.

30. Leytin V. Apoptosis in the anucleate platelet. Blood Rev. 2012;26(2):51-63.

31. Ravichandran KS, Lorenz U. Engulfment of apoptotic cells: signals for a good meal. Nat Rev Immunol. 2007;7(12):964-974.

32. Elliott MR, Ravichandran KS. The dynamics of apoptotic cell clearance. Dev Cell. 2016;38(2):147-160.

33. Moujalled D, et al. Cyclic-AMP-dependent protein kinase A regulates apoptosis by stabilizing the BH3-only protein Bim. EMBO Rep. 2011;12(1):77-83.

34. Naderi EH, Jochemsen AG, Blomhoff HK, Naderi S. Activation of cAMP signaling interferes with stress-induced p53 accumulation in ALL-derived cells by promoting the interaction between $\mathrm{p} 53$ and HDM2. Neoplasia. 2011;13(7):653-663.

35. Mihara $M$, et al. $p 53$ has a direct apoptogenic role at the mitochondria. Mol Cell.2003;11(3):577-590.

36. Datta SR, et al. 14-3-3 proteins and survival kinases cooperate to inactivate $\mathrm{BAD}$ by $\mathrm{BH} 3$ domain phosphorylation. Mol Cell.2000;6(1):41-51.

37. Lizcano JM, Morrice N, Cohen P. Regulation of $\mathrm{BAD}$ by cAMP-dependent protein kinase is mediated via phosphorylation of a novel site, Ser155. Biochem J. 2000;349(Pt 2):547-557.

38. Yang E, Zha J, Jockel J, Boise LH, Thompson CB, Korsmeyer SJ. Bad, a heterodimeric partner for
Bcl-XL and Bcl-2, displaces Bax and promotes cell death. Cell.1995;80(2):285-291.

39. Zha J, Harada H, Yang E, Jockel J, Korsmeyer SJ. Serine phosphorylation of death agonist BAD in response to survival factor results in binding to 14-3-3 not BCL-X(L). Cell. 1996;87(4):619-628.

40. Nieswandt B, Bergmeier W, Rackebrandt K, Gessner JE, Zirngibl H. Identification of critical antigen-specific mechanisms in the development of immune thrombocytopenic purpura in mice. Blood. 2000;96(7):2520-2527.

41. Grozovsky R, Giannini S, Falet H, Hoffmeister KM. Regulating billions of blood platelets: glycans and beyond. Blood. 2015;126(16):1877-1884 .

42. Li J, et al. Desialylation is a mechanism of Fcindependent platelet clearance and a therapeutic target in immune thrombocytopenia. Nat Commun. 2015;6:7737.

43. Dutta-Roy AK, Sinha AK. Purification and properties of prostaglandin E1/prostacyclin receptor of human blood platelets. J Biol Chem. 1987;262(26):12685-12691.

44. Lucas FV, Skrinska VA, Chisolm GM, Hesse BL. Stability of prostacyclin in human and rabbit whole blood and plasma. Thromb Res. 1986;43(4):379-387.

45. Adams JM, et al. Subversion of the Bcl-2 life/death switch in cancer development and therapy. Cold Spring Harb Symp Quant Biol. 2005;70:469-477.

46. Canault M, et al. p38 mitogen-activated protein kinase activation during platelet storage: consequences for platelet recovery and hemostatic function in vivo. Blood. 2010;115(9):1835-1842.

47. Chen W, et al. Inhibiting GPIb $\alpha$ shedding preserves post-transfusion recovery and hemostatic function of platelets after prolonged storage. Arterioscler Thromb Vasc Biol. 2016;36(9):1821-1828.

48. van der Wal DE, et al. Arachidonic acid depletion extends survival of cold-stored platelets by interfering with the [glycoprotein Ib $\alpha-14-3-3 \zeta$ ] association. Haematologica. 2012;97(10):1514-1522.

49. Gitz E, et al. Improved platelet survival after cold storage by prevention of glycoprotein Iba clustering in lipid rafts. Haematologica. 2012;97(12):1873-1881.

50. van der Wal DE, Du VX, Lo KS, Rasmussen JT, Verhoef S, Akkerman JW. Platelet apoptosis by cold-induced glycoprotein Ib $\alpha$ clustering. J Thromb Haemost. 2010;8(11):2554-2562.

51. Kramer RM, et al. p38 mitogen-activated protein kinase phosphorylates cytosolic phospholipase A2 (cPLA2) in thrombin-stimulated platelets. Evidence that proline-directed phosphorylation is not required for mobilization of arachidonic acid by cPLA2. J Biol Chem. 1996;271(44):27723-27729.

52. Stasi R, Evangelista ML, Stipa E, Buccisano F, Venditti A, Amadori S. Idiopathic thrombocytopenic purpura: current concepts in pathophysiology and management. Thromb Haemost. 2008;99(1):4-13.

53. Ranger AM, et al. Bad-deficient mice develop diffuse large B cell lymphoma. Proc Natl Acad Sci US A. 2003;100(16):9324-9329. 